\title{
Twin solutions to semipositone boundary value problems for fractional differential equations with coupled integral boundary conditions
}

\author{
Daliang Zhao, Yansheng Liu* \\ School of Mathematics and Statistics, Shandong Normal University, Jinan, 250014, P. R. China.
}

Communicated by N. Hussain

\begin{abstract}
This paper investigates the existence of at least two positive solutions for the following high-order fractional semipositone boundary value problem (SBVP, for short) with coupled integral boundary value conditions:

$$
\left\{\begin{array}{l}
D_{0^{+}}^{\alpha} u(t)+\lambda f(t, u(t), v(t))=0, \quad t \in(0,1), \\
D_{0^{+}}^{\alpha} v(t)+\lambda g(t, u(t), v(t))=0, \quad t \in(0,1), \\
u^{(j)}(0)=v^{(j)}(0)=0, j=0,1,2, \cdots, n-2, \\
D_{0^{+}}^{\alpha-1} u(1)=\lambda_{1} \int_{0}^{\eta_{1}} v(t) d t, \\
D_{0^{+}}^{\alpha-1} v(1)=\lambda_{2} \int_{0}^{\eta_{2}} u(t) d t,
\end{array}\right.
$$

where $n-1<\alpha \leqslant n, n \geqslant 3,0<\eta_{1}, \eta_{2} \leqslant 1, \lambda, \lambda_{1}, \lambda_{2}$ are parameters and satisfy $\lambda_{1} \lambda_{2}\left(\eta_{1} \eta_{2}\right)^{\alpha}<\Gamma^{2}(\alpha+1)$, D $0^{+}$is the standard Riemann-Liouville derivative, and $f, g$ are continuous and semipositone. By using the nonlinear alternative of Leray-Schauder type, Krasnoselskii's fixed point theorems, and the theory of fixed point index on cone, we establish some existence results of multiple positive solutions to the considered fractional SBVP. As applications, two examples are presented to illustrate our main results. (C)2017 All rights reserved.
\end{abstract}

Keywords: Fractional differential equations, semipositone boundary value problem, coupled integral boundary value conditions, fixed point index.

2010 MSC: 34A08, 34B15, 34B18.

\section{Introduction}

Fractional calculus and fractional differential equations have been investigated extensively due to their numerous and frequent applications in various fields of engineering and scientific disciplines such as physics, chemistry, economics, control theory, aerodynamics and electromagnetics, etc. Fractional derivatives provide an excellent tool for the description of memory and hereditary properties of various materials and processes. Thus fractional models are the natural substitutes of the classical integer-order model. For an extensive collection of such theory, methods and applications, we refer the readers to the materials $[1,3,9,10,13,14,16,18,21-27]$ and references therein.

\footnotetext{
*Corresponding author

Email addresses: dlzhao928@sdnu.edu.cn (Daliang Zhao), ysliu@sdnu.edu.cn (Yansheng Liu)
} 
Some new types of fractional derivatives are also reported in several excellent papers. For example, in [31], Yang introduced a class of the fractional derivatives of constant and variable orders for the first time. Fractional-order relaxation equations of constants and variable orders in the sense of Caputo type were modeled from mathematical view of point and they were very efficient in description of the complex phenomenon arising in heat transfer. In [32], the authors proposed a new fractional derivative without singular kernel. This was an extension of the Riemann-Liouville fractional derivative with singular kernel and had some important applications in the modeling of the fractional-order heat flow. In addition, a new definition of the local fractional derivative (LFD) was formulated to describe some non-differentiable problems that occur in fractal engineering recently. In [33], the authors studied the LC-electric circuit with the non-differentiable inductor and capacitor elements with the help of LFDs and fractal electrodynamics. They gave the comparative results among LFD, Riemann-Liouville fractional derivative and conventional derivative, which indicated that LFD was a new tool suitable for the study of a large class of electric circuits, and they had opened some new perspectives towards the characterization of non-differentiable electric circuits via LFDs.

On the other hand, coupled boundary conditions arise in the research of Sturm-Liouville problems and reaction-diffusion equations, see [15] for a good overview, and have wide applications in various fields such as engineering and sciences. While problems with integral boundary conditions arise in thermal conduction problems, semiconductor problems and hydrodynamic problems.

For example, in [28], by means of the method of upper and lower solutions and the Schauder fixed point theorem, Vong investigated the positive solutions of nonlocal BVP for a class of fractional differential equations:

$$
\left\{\begin{array}{l}
{ }^{C} D_{0^{+}}^{\alpha} x(t)+f(t, x(t))=0,0<t<1, \\
x^{\prime}(0)=\cdots=x^{(n-1)}(0)=0, x(1)=\int_{0}^{1} x(s) d \mu(s),
\end{array}\right.
$$

where $n \geqslant 2, \alpha \in(n-1, n)$ and $\mu(s)$ is a function of bounded variation.

In [4], Asif and Khan considered the following BVP:

$$
\left\{\begin{array}{l}
-u^{\prime \prime}(t)=f(t, u(t), v(t)), \quad t \in(0,1), \\
-v^{\prime \prime}(t)=g(t, u(t), v(t)), \quad t \in(0,1), \\
u(0)=0, u(1)=\alpha v(\xi), \\
v(0)=0, v(1)=\beta u(\eta),
\end{array}\right.
$$

where $f, g:(0,1) \times[0,+\infty) \times[0,+\infty) \rightarrow[0,+\infty)$ are continuous and singular at $t=0, t=1$, and parameters $\alpha, \beta, \xi, \eta$ satisfy $\xi, \eta \in(0,1), 0<\alpha \beta \xi \eta<1$. They obtained the existence of at least one positive solution by using Krasnoselskii's fixed point theorem in a special cone.

In [7], Goodrich investigated a system of fractional BVP given by

$$
\left\{\begin{array}{l}
-D_{0_{1}^{+}}^{v_{1}} u_{1}(t)=\lambda_{1} a_{1}(t) f\left(u_{1}(t), u_{2}(t)\right), 0<t<1, \\
-D_{0_{2}^{+}}^{v_{2}} u_{2}(t)=\lambda_{2} a_{2}(t) g\left(u_{1}(t), u_{2}(t)\right), \\
u_{1}^{(i)}(0)=u_{2}^{(i)}(0)=0,0 \leqslant i \leqslant n-2, \\
{\left[D_{0^{+}}^{\alpha} u_{1}(t)\right]_{t=1}=\phi_{1}(u),\left[D_{0^{+}}^{\alpha} u_{2}(t)\right]_{t=1}=\phi_{2}(u), \quad 1 \leqslant \alpha \leqslant n-2 .}
\end{array}\right.
$$

The author gave the existence of at least one positive solution in view of Krasnoselskii's fixed point theorem under the local and the nonlocal boundary conditions, respectively.

Motivated by the above-mentioned works, we aim to establish some existence criteria of multiple positive solutions for the following high-order semipositone fractional differential system with coupled integral boundary conditions:

$$
\left\{\begin{array}{l}
D_{0^{+}}^{\alpha} u(t)+\lambda f(t, u(t), v(t))=0, \quad t \in(0,1), \\
D_{0^{+}}^{\alpha} v(t)+\lambda g(t, u(t), v(t))=0, \quad t \in(0,1) \\
u^{(j)}(0)=v^{(j)}(0)=0, j=0,1,2, \cdots, n-2, \\
D_{0^{+}}^{\alpha-1} u(1)=\lambda_{1} \int_{0}^{\eta_{1}} v(t) d t \\
D_{0^{+}}^{\alpha-1} v(1)=\lambda_{2} \int_{0}^{\eta_{2}} u(t) d t
\end{array}\right.
$$


where $n-1<\alpha \leqslant n, n \geqslant 3,0<\eta_{1}, \eta_{2} \leqslant 1, \lambda, \lambda_{1}, \lambda_{2}$ are parameters and satisfy $\lambda_{1} \lambda_{2}\left(\eta_{1} \eta_{2}\right)^{\alpha}<\Gamma^{2}(\alpha+1)$, $\mathrm{D}_{0^{+}}^{\alpha}$ is the standard Riemann-Liouville derivative, and $f, g$ are sign-changing continuous functions.

The main features of the present paper are as follows. Firstly, the nonlinear terms we discuss here are semipositone. This is different from lots of the previous articles, see $[5,6,17,19,20,29]$ and the reference therein, where the nonlinearity needs to be nonnegative to get the positive solutions. Secondly, the boundary value conditions of the considered system are fractional high-order coupled integral boundary value conditions and contain parameters, and this enriches the theoretical knowledge of above mentioned works. Thirdly, the given conditions $\widehat{\mathrm{f}}_{0}, \widehat{\mathrm{g}}_{0}$ and $(\mathrm{H} 4)$ are quite different from many other papers such as $[11,34,35]$, and then the methods used in Theorem 3.2 are also different from these papers.

In this paper, we derive corresponding integral representation for SBVP (1.1) and give some important properties which will play an important role in our proof. This is our key to establish a cone. By utilizing the nonlinear alternative of Leray-Schauder type, Krasnoselskii's fixed point theorems, and the theory of fixed point index on cone, we derive an interval of parameter $\lambda$ such that for any $\lambda$ lying in this interval, the SBVP (1.1) has at least two positive solutions. To the best of our knowledge, no contribution exists considering the multiple positive solutions for SBVP (1.1). It should also be noted that $\eta_{1}, \eta_{2} \in(0,1]$ are more general than those in $[28,30,35,36]$, in which $\eta_{1}, \eta_{2}$ equal to one. By a positive solution of SBVP (1.1) we mean a pair of functions $(u, v) \in C[0,1] \times C[0,1]$ satisfying (1.1) with $D_{0^{+}}^{\alpha} u(t), D_{0^{+}}^{\alpha} v(t) \in L(0,1)$ and $u(t)>0, v(t)>0$ for any $t \in(0,1]$.

The rest of this paper is organized as follows. Section 2 contains some necessary preliminaries and lemmas. Section 3 investigates the existence of multiple positive solutions to system (1.1). Finally, in Section 4, two illustrative examples are presented to demonstrate our new results.

\section{Preliminaries and lemmas}

For the convenience of readers, we first recall some well-known results about the standard RiemannLiouville derivative. For details, please refer to $[22,25,26]$ and the references therein.

Definition 2.1. The Riemann-Liouville standard fractional integral of order $\alpha>0$ of a continuous function $u:(0,+\infty) \rightarrow \mathbb{R}$ is given by

$$
\mathrm{I}_{0^{+}}^{\alpha} \mathrm{u}(\mathrm{t})=\frac{1}{\Gamma(\alpha)} \int_{0}^{\mathrm{t}}(\mathrm{t}-\mathrm{s})^{\alpha-1} \mathrm{u}(\mathrm{s}) \mathrm{ds},
$$

provided that the right side integral is pointwise defined on $(0,+\infty)$.

Definition 2.2. The Riemann-Liouville fractional derivative of order $\alpha$ of a continuous function $u$ : $(0,+\infty) \rightarrow \mathbb{R}$ is defined by

$$
D_{0+}^{\alpha} u(t)=\frac{1}{\Gamma(n-\alpha)}\left(\frac{d}{d t}\right)^{n} \int_{0}^{t} \frac{u(s)}{(t-s)^{\alpha-n+1}} d s,
$$

where $n=[\alpha]+1$, provided that the right side is pointwise defined on $(0,+\infty)$.

As an example, for $\varpi>-1$, we have $D_{0+}^{\alpha} u^{\varpi}=\frac{\Gamma(1+\varpi)}{\Gamma(1+\varpi-\alpha)} u^{\varpi-\alpha}$.

Lemma 2.3 ([12]). Let $\alpha>0$. Then the following equality holds for $u \in \mathrm{L}(0,1), \mathrm{D}_{0^{+}}^{\alpha} \in \mathrm{L}(0,1)$,

$$
\mathrm{I}_{0^{+}}^{\alpha} \mathrm{D}_{0^{+}}^{\alpha} \mathrm{u}(\mathrm{t})=\mathrm{u}(\mathrm{t})+\mathrm{c}_{1} \mathrm{t}^{\alpha-1}+\mathrm{c}_{2} \mathrm{t}^{\alpha-2}+\cdots+\mathrm{c}_{\mathrm{n}} \mathrm{t}^{\alpha-n},
$$

where $c_{i} \in \mathbb{R}, i=1,2, \cdots, n$, and $n-1<\alpha \leqslant n$.

For convenience in the following discussion, we set

$$
k_{1}=\frac{\eta_{1}^{\alpha}}{\Gamma(\alpha+1)}, \quad k_{2}=\frac{\eta_{2}^{\alpha}}{\Gamma(\alpha+1)} .
$$

It is obvious that $1-k_{1} k_{2} \lambda_{1} \lambda_{2}>0$ if $\lambda_{1} \lambda_{2}\left(\eta_{1} \eta_{2}\right)^{\alpha}<\Gamma^{2}(\alpha+1)$. 
Lemma 2.4. Let $\mathrm{x}, \mathrm{y} \in \mathrm{C}[0,1]$ be given functions. Then the unique solution of system

$$
\left\{\begin{array}{l}
D_{0^{+}}^{\alpha} u(t)+x(t)=0, \quad t \in(0,1) \\
D_{0^{+}}^{\alpha} v(t)+y(t)=0, \quad t \in(0,1) \\
u^{(j)}(0)=v^{(j)}(0)=0, \quad j=0,1,2, \cdots, n-2, \\
D_{0^{+}}^{\alpha-1} u(1)=\lambda_{1} \int_{0}^{\eta_{1}} v(t) d t \\
D_{0^{+}}^{\alpha-1} v(1)=\lambda_{2} \int_{0}^{\eta_{2}} u(t) d t
\end{array}\right.
$$

where $n-1<\alpha \leqslant n, n \geqslant 3,0<\eta_{1}, \eta_{2} \leqslant 1, \lambda_{1} \lambda_{2}\left(\eta_{1} \eta_{2}\right)^{\alpha}<\Gamma^{2}(\alpha+1)$, is given by

$$
u(t)=\int_{0}^{1} G_{1}(t, s) x(s) d s+\int_{0}^{1} H_{1}(t, s) y(s) d s, \quad v(t)=\int_{0}^{1} G_{2}(t, s) y(s) d s+\int_{0}^{1} H_{2}(t, s) x(s) d s,
$$

where

$$
\begin{aligned}
& G_{1}(t, s)= \begin{cases}\frac{k_{1} \lambda_{1} \lambda_{2} t^{\alpha-1}}{\left(1-k_{1} k_{2} \lambda_{1} \lambda_{2}\right) \Gamma(\alpha)} \int_{0}^{1} G(\tau, s) d \tau+\frac{t^{\alpha-1}-(t-s)^{\alpha-1}}{\Gamma(\alpha)}, & 0 \leqslant s \leqslant \min \left\{\eta_{2}, t\right\}, \\
\frac{k_{1} \lambda_{1} \lambda_{2} t^{\alpha-1}}{\left(1-k_{1} k_{2} \lambda_{1} \lambda_{2}\right) \Gamma(\alpha)} \int_{0}^{1} G(\tau, s) d \tau+\frac{t^{\alpha-1}}{\Gamma(\alpha)}, & t \leqslant s \leqslant \eta_{2}, \\
\frac{t^{\alpha-1}-(t-s)^{\alpha-1}}{\Gamma(\alpha)}, & \eta_{2}<s \leqslant t, \\
\frac{t^{\alpha-1}}{\Gamma(\alpha)}, & s \geqslant \max \left\{\eta_{2}, t\right\},\end{cases} \\
& G_{2}(t, s)= \begin{cases}\frac{k_{2} \lambda_{1} \lambda_{2} t^{\alpha-1}}{\left(1-k_{1} k_{2} \lambda_{1} \lambda_{2}\right) \Gamma(\alpha)} \int_{0}^{1} G(\tau, s) d \tau+\frac{t^{\alpha-1}-(t-s)^{\alpha-1}}{\Gamma(\alpha)}, & 0 \leqslant s \leqslant \min \left\{\eta_{1}, t\right\}, \\
\frac{k_{2} \lambda_{1} \lambda_{2} t^{\alpha-1}}{\left(1-k_{1} k_{2} \lambda_{1} \lambda_{2}\right) \Gamma(\alpha)} \int_{0}^{1} G(\tau, s) d \tau+\frac{t^{\alpha-1}}{\Gamma(\alpha)}, & t \leqslant s \leqslant \eta_{1}, \\
\frac{t^{\alpha-1}-(t-s)^{\alpha-1}}{\Gamma(\alpha)}, & \eta_{1}<s \leqslant t, \\
\frac{t^{\alpha-1}}{\Gamma(\alpha)}, & s \geqslant \max \left\{\eta_{1}, t\right\},\end{cases} \\
& H_{1}(t, s)=\frac{\lambda_{1} t^{\alpha-1}}{\left(1-k_{1} k_{2} \lambda_{1} \lambda_{2}\right) \Gamma(\alpha)} \int_{0}^{\eta_{1}} G(\tau, s) d \tau \text {, } \\
& \mathrm{H}_{2}(\mathrm{t}, \mathrm{s})=\frac{\lambda_{2} \mathrm{t}^{\alpha-1}}{\left(1-\mathrm{k}_{1} \mathrm{k}_{2} \lambda_{1} \lambda_{2}\right) \Gamma(\alpha)} \int_{0}^{\eta_{2}} \mathrm{G}(\tau, \mathrm{s}) \mathrm{d} \tau, \\
& G(t, s)= \begin{cases}\frac{t^{\alpha-1}-(t-s)^{\alpha-1}}{\Gamma(\alpha)}, & s \leqslant t \\
\frac{t^{\alpha-1}}{\Gamma(\alpha)}, & s \geqslant t .\end{cases}
\end{aligned}
$$

Proof. In view of Lemma 2.3, we reduce (2.1) to the following equivalent equation

$$
\begin{aligned}
& u(t)=-I_{0^{+}}^{\alpha} x(t)+c_{1} t^{\alpha-1}+c_{2} t^{\alpha-2}+\cdots+c_{n} t^{\alpha-n}, \\
& v(t)=-I_{0^{+}}^{\alpha} y(t)+c_{1}^{\prime} t^{\alpha-1}+c_{2}^{\prime} t^{\alpha-2}+\cdots+c_{n}^{\prime} t^{\alpha-n}
\end{aligned}
$$

for some constants $c_{i}, c_{i}^{\prime} \in \mathbb{R}, i=1,2, \cdots, n$.

The boundary value conditions $\mathfrak{u}^{(\mathfrak{j})}(0)=v^{(j)}(0)=0, j=0,1,2, \cdots, n-2$ imply that $c_{i}=c_{i}^{\prime}=0, i=$ $2,3, \cdots, n$, and so

$$
u(t)=-\int_{0}^{t} \frac{(t-s)^{\alpha-1}}{\Gamma(\alpha)} x(s) d s+c_{1} t^{\alpha-1}, \quad v(t)=-\int_{0}^{t} \frac{(t-s)^{\alpha-1}}{\Gamma(\alpha)} y(s) d s+c_{1}^{\prime} t^{\alpha-1}
$$


Then, we have

$$
D_{0^{+}}^{\alpha-1} u(t)=-\int_{0}^{t} x(s) d s+c_{1} \Gamma(\alpha), \quad D_{0^{+}}^{\alpha-1} v(t)=-\int_{0}^{t} y(s) d s+c_{1}^{\prime} \Gamma(\alpha)
$$

Thus

$$
D_{0^{+}}^{\alpha-1} \mathfrak{u}(1)=-\int_{0}^{1} x(s) d s+c_{1} \Gamma(\alpha), \quad D_{0^{+}}^{\alpha-1} v(1)=-\int_{0}^{1} y(s) d s+c_{1}^{\prime} \Gamma(\alpha) .
$$

From the boundary conditions $D_{0^{+}}^{\alpha-1} u(1)=\lambda_{1} \int_{0}^{\eta_{1}} v(t) d t$ and $D_{0^{+}}^{\alpha-1} v(1)=\lambda_{2} \int_{0}^{\eta_{2}} u(t) d t$, we get

$$
c_{1}=\frac{1}{\Gamma(\alpha)}\left(\lambda_{1} \int_{0}^{\eta_{1}} v(t) d t+\int_{0}^{1} x(s) d s\right), \quad c_{1}^{\prime}=\frac{1}{\Gamma(\alpha)}\left(\lambda_{2} \int_{0}^{\eta_{2}} u(t) d t+\int_{0}^{1} y(s) d s\right) .
$$

So, we obtain

$$
\begin{aligned}
u(t) & =-\int_{0}^{t} \frac{(t-s)^{\alpha-1}}{\Gamma(\alpha)} x(s) d s+\frac{\lambda_{1}}{\Gamma(\alpha)} t^{\alpha-1} \int_{0}^{\eta_{1}} v(t) d t+\frac{1}{\Gamma(\alpha)} t^{\alpha-1} \int_{0}^{1} x(s) d s \\
& =\int_{0}^{1} G(t, s) x(s) d s+\frac{t^{\alpha-1}}{\Gamma(\alpha)} D_{0^{+}}^{\alpha-1} u(1), \\
v(t) & =-\int_{0}^{t} \frac{(t-s)^{\alpha-1}}{\Gamma(\alpha)} y(s) d s+\frac{\lambda_{2}}{\Gamma(\alpha)} t^{\alpha-1} \int_{0}^{\eta_{2}} u(t) d t+\frac{1}{\Gamma(\alpha)} t^{\alpha-1} \int_{0}^{1} y(s) d s \\
& =\int_{0}^{1} G(t, s) y(s) d s+\frac{t^{\alpha-1}}{\Gamma(\alpha)} D_{0^{+}}^{\alpha-1} v(1) .
\end{aligned}
$$

Integrating (2.7) and (2.8) with respect to $t$ on $\left[0, \eta_{2}\right]$ and $\left[0, \eta_{1}\right]$ respectively gives

$$
\int_{0}^{\eta_{2}} u(t) d t=k_{2} D_{0^{+}}^{\alpha-1} u(1)+\int_{0}^{\eta_{2}} \int_{0}^{1} G(t, s) x(s) d s d t, \quad \int_{0}^{\eta_{1}} v(t) d t=k_{1} D_{0^{+}}^{\alpha-1} v(1)+\int_{0}^{\eta_{1}} \int_{0}^{1} G(t, s) y(s) d s d t .
$$

By simple calculations, it follows that

$$
\begin{aligned}
& D_{0^{+}}^{\alpha-1} u(1)=\frac{1}{1-k_{1} k_{2} \lambda_{1} \lambda_{2}}\left(k_{1} \lambda_{1} \lambda_{2} \int_{0}^{\eta_{2}} \int_{0}^{1} G(t, s) x(s) d s d t+\lambda_{1} \int_{0}^{\eta_{1}} \int_{0}^{1} G(t, s) y(s) d s d t\right) \\
& D_{0^{+}}^{\alpha-1} v(1)=\frac{1}{1-k_{1} k_{2} \lambda_{1} \lambda_{2}}\left(\lambda_{2} \int_{0}^{\eta_{2}} \int_{0}^{1} G(t, s) x(s) d s d t+k_{2} \lambda_{1} \lambda_{2} \int_{0}^{\eta_{1}} \int_{0}^{1} G(t, s) y(s) d s d t\right)
\end{aligned}
$$

Substituting (2.9) and (2.10) into (2.7) and (2.8), respectively, we have

$$
\begin{aligned}
u(t)= & \int_{0}^{\eta_{2}} \int_{0}^{1} \frac{k_{1} \lambda_{1} \lambda_{2} t^{\alpha-1}}{\left(1-k_{1} k_{2} \lambda_{1} \lambda_{2}\right) \Gamma(\alpha)} G(\tau, s) x(s) d s d \tau \\
& +\int_{0}^{\eta_{1}} \int_{0}^{1} \frac{\lambda_{1} t^{\alpha-1}}{\left(1-k_{1} k_{2} \lambda_{1} \lambda_{2}\right) \Gamma(\alpha)} G(\tau, s) y(s) d s d \tau+\int_{0}^{1} G(t, s) x(s) d s, \\
v(t)= & \int_{0}^{\eta_{2}} \int_{0}^{1} \frac{\lambda_{2} t^{\alpha-1}}{\left(1-k_{1} k_{2} \lambda_{1} \lambda_{2}\right) \Gamma(\alpha)} G(\tau, s) x(s) d s d \tau \\
& +\int_{0}^{\eta_{1}} \int_{0}^{1} \frac{k_{2} \lambda_{1} \lambda_{2} t^{\alpha-1}}{\left(1-k_{1} k_{2} \lambda_{1} \lambda_{2}\right) \Gamma(\alpha)} G(\tau, s) y(s) d s d \tau+\int_{0}^{1} G(t, s) y(s) d s .
\end{aligned}
$$

In the following, we divide the proof into four cases. 
Case $1 . t \leqslant \eta_{2}$.

$$
\begin{aligned}
u(t)= & \left(\int_{0}^{t}+\int_{t}^{\eta_{2}}\right) \int_{0}^{1} \frac{k_{1} \lambda_{1} \lambda_{2} t^{\alpha-1}}{\left(1-k_{1} k_{2} \lambda_{1} \lambda_{2}\right) \Gamma(\alpha)} G(\tau, s) x(s) d s d \tau \\
& +\int_{0}^{1}\left(\frac{\lambda_{1} t^{\alpha-1}}{\left(1-k_{1} k_{2} \lambda_{1} \lambda_{2}\right) \Gamma(\alpha)} \int_{0}^{\eta_{1}} G(\tau, s) d \tau\right) y(s) d s+\left(\int_{0}^{t}+\int_{t}^{\eta_{2}}+\int_{\eta_{2}}^{1}\right) G(t, s) x(s) d s \\
= & \int_{0}^{t}\left(\frac{k_{1} \lambda_{1} \lambda_{2} t^{\alpha-1}}{\left(1-k_{1} k_{2} \lambda_{1} \lambda_{2}\right) \Gamma(\alpha)} \int_{0}^{1} G(\tau, s) d \tau+\frac{t^{\alpha-1}-(t-s)^{\alpha-1}}{\Gamma(\alpha)}\right) x(s) d s \\
& +\int_{t}^{\eta_{2}}\left(\frac{k_{1} \lambda_{1} \lambda_{2} t^{\alpha-1}}{\left(1-k_{1} k_{2} \lambda_{1} \lambda_{2}\right) \Gamma(\alpha)} \int_{0}^{1} G(\tau, s) d \tau+\frac{t^{\alpha-1}}{\Gamma(\alpha)}\right) x(s) d s+\int_{\eta_{2}}^{1} \frac{t^{\alpha-1}}{\Gamma(\alpha)} x(s) d s \\
& +\int_{0}^{1}\left(\frac{\lambda_{1} t^{\alpha-1}}{\left(1-k_{1} k_{2} \lambda_{1} \lambda_{2}\right) \Gamma(\alpha)} \int_{0}^{\eta_{1}} G(\tau, s) d \tau\right) y(s) d s \\
= & \int_{0}^{1} G_{1}(t, s) x(s) d s+\int_{0}^{1} H_{1}(t, s) y(s) d s .
\end{aligned}
$$

Case 2. $t \geqslant \eta_{2}$.

$$
\begin{aligned}
u(t)= & \left(\int_{0}^{\eta_{2}}+\int_{\eta_{2}}^{t}\right) \int_{0}^{1} \frac{k_{1} \lambda_{1} \lambda_{2} t^{\alpha-1}}{\left(1-k_{1} k_{2} \lambda_{1} \lambda_{2}\right) \Gamma(\alpha)} G(\tau, s) x(s) d s d \tau \\
& +\int_{0}^{1}\left(\frac{\lambda_{1} t^{\alpha-1}}{\left(1-k_{1} k_{2} \lambda_{1} \lambda_{2}\right) \Gamma(\alpha)} \int_{0}^{\eta_{1}} G(\tau, s) d \tau\right) y(s) d s+\left(\int_{0}^{\eta_{2}}+\int_{\eta_{2}}^{t}+\int_{t}^{1}\right) G(t, s) x(s) d s \\
= & \int_{0}^{\eta_{2}}\left(\frac{k_{1} \lambda_{1} \lambda_{2} t^{\alpha-1}}{\left(1-k_{1} k_{2} \lambda_{1} \lambda_{2}\right) \Gamma(\alpha)} \int_{0}^{1} G(\tau, s) d \tau+\frac{t^{\alpha-1}-(t-s)^{\alpha-1}}{\Gamma(\alpha)}\right) x(s) d s \\
& +\int_{\eta_{2}}^{t} \frac{t^{\alpha-1}-(t-s)^{\alpha-1}}{\Gamma(\alpha)} x(s) d s+\int_{t}^{1} \frac{t^{\alpha-1}}{\Gamma(\alpha)} x(s) d s+\int_{0}^{1}\left(\frac{\lambda_{1} t^{\alpha-1}}{\left(1-k_{1} k_{2} \lambda_{1} \lambda_{2}\right) \Gamma(\alpha)} \int_{0}^{\eta_{1}} G(\tau, s) d \tau\right) y(s) d s \\
= & \int_{0}^{1} G_{1}(t, s) x(s) d s+\int_{0}^{1} H_{1}(t, s) y(s) d s .
\end{aligned}
$$

Case 3. $t \leqslant \eta_{1}$.

$$
\begin{aligned}
v(t)= & \left(\int_{0}^{t}+\int_{t}^{\eta_{1}}\right) \int_{0}^{1} \frac{k_{2} \lambda_{1} \lambda_{2} t^{\alpha-1}}{\left(1-k_{1} k_{2} \lambda_{1} \lambda_{2}\right) \Gamma(\alpha)} G(\tau, s) y(s) d s d \tau \\
& +\int_{0}^{1}\left(\frac{\lambda_{2} t^{\alpha-1}}{\left(1-k_{1} k_{2} \lambda_{1} \lambda_{2}\right) \Gamma(\alpha)} \int_{0}^{\eta_{2}} G(\tau, s) d \tau\right) x(s) d s+\left(\int_{0}^{t}+\int_{t}^{\eta_{1}}+\int_{\eta_{1}}^{1}\right) G(t, s) y(s) d s \\
= & \int_{0}^{t}\left(\frac{k_{2} \lambda_{1} \lambda_{2} t^{\alpha-1}}{\left(1-k_{1} k_{2} \lambda_{1} \lambda_{2}\right) \Gamma(\alpha)} \int_{0}^{1} G(\tau, s) d \tau+\frac{t^{\alpha-1}-(t-s)^{\alpha-1}}{\Gamma(\alpha)}\right) y(s) d s \\
& +\int_{t}^{\eta_{1}}\left(\frac{k_{2} \lambda_{1} \lambda_{2} t^{\alpha-1}}{\left(1-k_{1} k_{2} \lambda_{1} \lambda_{2}\right) \Gamma(\alpha)} \int_{0}^{1} G(\tau, s) d \tau+\frac{t^{\alpha-1}}{\Gamma(\alpha)}\right) y(s) d s+\int_{\eta_{1}}^{1} \frac{t^{\alpha-1}}{\Gamma(\alpha)} y(s) d s \\
& +\int_{0}^{1}\left(\frac{\lambda_{2} t^{\alpha-1}}{\left(1-k_{1} k_{2} \lambda_{1} \lambda_{2}\right) \Gamma(\alpha)} \int_{0}^{\eta_{2}} G(\tau, s) d \tau\right) x(s) d s \\
= & \int_{0}^{1} G_{2}(t, s) y(s) d s+\int_{0}^{1} H_{2}(t, s) x(s) d s .
\end{aligned}
$$

Case 4. $t \geqslant \eta_{1}$.

$$
v(t)=\left(\int_{0}^{\eta_{1}}+\int_{\eta_{1}}^{t}\right) \int_{0}^{1} \frac{k_{2} \lambda_{1} \lambda_{2} t^{\alpha-1}}{\left(1-k_{1} k_{2} \lambda_{1} \lambda_{2}\right) \Gamma(\alpha)} G(\tau, s) y(s) d s d \tau
$$




$$
\begin{aligned}
& +\int_{0}^{1}\left(\frac{\lambda_{2} t^{\alpha-1}}{\left(1-k_{1} k_{2} \lambda_{1} \lambda_{2}\right) \Gamma(\alpha)} \int_{0}^{\eta_{2}} G(\tau, s) d \tau\right) x(s) d s+\left(\int_{0}^{\eta_{1}}+\int_{\eta_{1}}^{t}+\int_{t}^{1}\right) G(t, s) y(s) d s \\
= & \int_{0}^{\eta_{1}}\left(\frac{k_{2} \lambda_{1} \lambda_{2} t^{\alpha-1}}{\left(1-k_{1} k_{2} \lambda_{1} \lambda_{2}\right) \Gamma(\alpha)} \int_{0}^{1} G(\tau, s) d \tau+\frac{t^{\alpha-1}-(t-s)^{\alpha-1}}{\Gamma(\alpha)}\right) y(s) d s \\
& +\int_{\eta_{1}}^{t} \frac{t^{\alpha-1}-(t-s)^{\alpha-1}}{\Gamma(\alpha)} y(s) d s+\int_{t}^{1} \frac{t^{\alpha-1}}{\Gamma(\alpha)} y(s) d s+\int_{0}^{1}\left(\frac{\lambda_{2} t^{\alpha-1}}{\left(1-k_{1} k_{2} \lambda_{1} \lambda_{2}\right) \Gamma(\alpha)} \int_{0}^{\eta_{2}} G(\tau, s) d \tau\right) x(s) d s \\
= & \int_{0}^{1} G_{2}(t, s) y(s) d s+\int_{0}^{1} H_{2}(t, s) x(s) d s .
\end{aligned}
$$

This completes the proof.

Lemma 2.5. The function $\mathrm{G}(\mathrm{t}, \mathrm{s})$ defined by (2.6) has the following property:

$$
\frac{\mathrm{t}^{\alpha-1}}{\Gamma(\alpha)}\left(1-(1-\mathrm{s})^{\alpha-1}\right) \leqslant \mathrm{G}(\mathrm{t}, \mathrm{s}) \leqslant \frac{1}{\Gamma(\alpha)}\left(1-(1-\mathrm{s})^{\alpha-1}\right) .
$$

Proof. For $s \leqslant t$, we have

$$
G(t, s)=\frac{t^{\alpha-1}-(t-s)^{\alpha-1}}{\Gamma(\alpha)}=\frac{t^{\alpha-1}}{\Gamma(\alpha)}\left(1-\left(1-\frac{s}{t}\right)^{\alpha-1}\right) \geqslant \frac{t^{\alpha-1}}{\Gamma(\alpha)}\left(1-(1-s)^{\alpha-1}\right) .
$$

From

$$
\frac{\partial G}{\partial t}(t, s)=\frac{(\alpha-1) t^{\alpha-2}-(\alpha-1)(t-s)^{\alpha-2}}{\Gamma(\alpha)} \geqslant 0
$$

it follows that

$$
G(t, s)=\frac{t^{\alpha-1}-(t-s)^{\alpha-1}}{\Gamma(\alpha)} \leqslant G(1, s)=\frac{1}{\Gamma(\alpha)}\left(1-(1-s)^{\alpha-1}\right) .
$$

For $s \geqslant t$, we know

$$
G(t, s)=\frac{t^{\alpha-1}}{\Gamma(\alpha)} \leqslant \frac{s^{\alpha-1}}{\Gamma(\alpha)} \leqslant \frac{1}{\Gamma(\alpha)}\left(1-(1-s)^{\alpha-1}\right)
$$

by means of $s^{\alpha-1}+(1-s)^{\alpha-1} \leqslant 1$ for $s \in(0,1]$.

On the other hand, $G(t, s)=\frac{t^{\alpha-1}}{\Gamma(\alpha)} \geqslant \frac{t^{\alpha-1}}{\Gamma(\alpha)}\left(1-(1-s)^{\alpha-1}\right)$ is obvious.

Summing up the above discussion, the conclusion of this lemma follows.

From Lemma 2.5 together with (2.2), (2.3), (2.4), and (2.5), we can obtain the following.

Lemma 2.6. The functions $\mathrm{G}_{1}(\mathrm{t}, \mathrm{s}), \mathrm{G}_{2}(\mathrm{t}, \mathrm{s})$ and $\mathrm{H}_{1}(\mathrm{t}, \mathrm{s}), \mathrm{H}_{2}(\mathrm{t}, \mathrm{s})$ defined respectively by (2.2), (2.3), (2.4), and (2.5) have the following properties:

(i) $v t^{\alpha-1}\left(1-(1-s)^{\alpha-1}\right) \leqslant G_{i}(t, s) \leqslant \rho\left(1-(1-s)^{\alpha-1}\right), i=1,2$;

(ii) $v t^{\alpha-1}\left(1-(1-s)^{\alpha-1}\right) \leqslant H_{i}(t, s) \leqslant \rho\left(1-(1-s)^{\alpha-1}\right), i=1,2$;

(iii) $G_{i}(t, s) \leqslant \rho t^{\alpha-1}, H_{i}(t, s) \leqslant \rho t^{\alpha-1}, i=1,2$,

where

$$
\begin{aligned}
& \rho=\max \left\{\frac{\max \left\{k_{1}, k_{2}\right\} \lambda_{1} \lambda_{2}}{\left(1-k_{1} k_{2} \lambda_{1} \lambda_{2}\right) \Gamma^{2}(\alpha)}+\frac{1}{\Gamma(\alpha)}, \frac{\max \left\{\lambda_{1} \eta_{1}, \lambda_{2} \eta_{2}\right\}}{\left(1-k_{1} k_{2} \lambda_{1} \lambda_{2}\right) \Gamma^{2}(\alpha)}\right\}, \\
& v=\min \left\{\frac{1}{\Gamma(\alpha)}, \frac{\min \left\{\lambda_{1} \eta_{1}^{\alpha}, \lambda_{2} \eta_{2}^{\alpha}\right\}}{\left(1-k_{1} k_{2} \lambda_{1} \lambda_{2}\right) \Gamma(\alpha) \Gamma(\alpha+1)}\right\} .
\end{aligned}
$$


Let $E=C[0,1]$ be the Banach space with the maximum norm $\|u\|=\max \{|u(t)|: t \in[0,1]\}$ for each $u \in E$. Obviously, $E \times E$ is a Banach space with the norm $\|(u, v)\|_{1}=\max \{\|u\|,\|v\|\}$. Denote

$$
P=\left\{(u, v) \in E \times E: u(t) \geqslant \gamma t^{\alpha-1}\|(u, v)\|_{1}, v(t) \geqslant \gamma t^{\alpha-1}\|(u, v)\|_{1}, t \in[0,1]\right\},
$$

where $\gamma=\frac{v}{\rho} \in(0,1)$. It is easy to verify that $P$ is a cone in $E \times E$. Let

$$
\mathrm{P}_{\mathrm{r}}=\left\{(\mathrm{u}, v) \in \mathrm{P}:\|(\mathrm{u}, v)\|_{1}<\mathrm{r}\right\}(\mathrm{r}>0) .
$$

Fix an interval $[\xi, \eta] \subset(0,1)$. Define several notations:

$$
\begin{aligned}
& f_{\infty}=\liminf _{\substack{u+v \rightarrow+\infty \\
u, v \geqslant 0}} \min _{\substack{t \in[\xi, \eta] \\
\text { ind }}} \frac{f(t, u, v)}{u+v},
\end{aligned}
$$

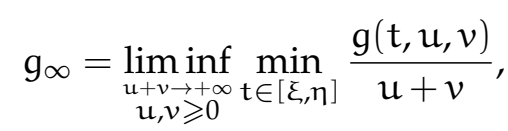

$$
\begin{aligned}
& \widehat{f_{0}}=\liminf _{\substack{u+v \rightarrow 0 \\
u, v \geqslant 0}} \min _{t \in[\xi, \eta]} f(t, u, v), \\
& \widehat{g}_{0}=\liminf _{\substack{u+v \rightarrow 0 \\
u, v \geqslant 0}} \min _{t \in[\xi, \eta]} g(t, u, v) .
\end{aligned}
$$

We present now the hypotheses that will be used in the sequel.

(H1) $f, g \in C([0,1] \times[0,+\infty) \times[0,+\infty),(-\infty,+\infty))$ and satisfy

$$
f(t, u, v) \geqslant-q_{1}(t), g(t, u, v) \geqslant-q_{2}(t), t \in(0,1), u, v \in[0,+\infty),
$$

where $\mathrm{q}_{\mathrm{i}} \in \mathrm{C}((0,1),[0,+\infty))$ and $0<\int_{0}^{1} \mathrm{q}_{\mathrm{i}}(\mathrm{s}) \mathrm{ds}<+\infty, i=1,2$.

$\left(H 1^{*}\right) f, g \in C((0,1) \times[0,+\infty) \times[0,+\infty),(-\infty,+\infty))$ and satisfy

$$
\begin{aligned}
& 0 \leqslant f(t, u, v)+q_{1}(t) \leqslant p_{1}(t) h_{1}(u, v), t \in(0,1), u, v \in[0,+\infty), \\
& 0 \leqslant g(t, u, v)+q_{2}(t) \leqslant p_{2}(t) h_{2}(u, v), t \in(0,1), u, v \in[0,+\infty),
\end{aligned}
$$

where $p_{i}, q_{i} \in C((0,1),[0,+\infty)), h_{i} \in C([0,+\infty) \times[0,+\infty),[0,+\infty))$ and

$$
0<\int_{0}^{1} p_{i}(s) d s<+\infty, 0<\int_{0}^{1} q_{i}(s) d s<+\infty, i=1,2 .
$$

(H2) $f(t, 0,0)>0, g(t, 0,0)>0$ for all $t \in[0,1]$.

(H3) $f_{\infty}=+\infty$ or $g_{\infty}=+\infty$.

(H4) $\widehat{f}_{0} \geqslant L$ or $\widehat{g}_{0} \geqslant L$, where $L>\frac{\int_{0}^{1}\left(q_{1}(s)+q_{2}(s)\right) d s}{\gamma^{2} \xi^{\alpha-1} \int_{\xi}^{\eta}\left(1-(1-s)^{\alpha-1}\right) d s}$.

We first consider the system of fractional BVP

$$
\left\{\begin{array}{l}
D_{0^{+}}^{\alpha} x(t)+\lambda\left[f\left(t,\left[x(t)-\omega_{1}(t)\right]^{*},\left[y(t)-\omega_{2}(t)\right]^{*}\right)+q_{1}(t)\right]=0, \quad t \in(0,1), \\
D_{0^{+}}^{\alpha} y(t)+\lambda\left[g\left(t,\left[x(t)-\omega_{1}(t)\right]^{*},\left[y(t)-\omega_{2}(t)\right]^{*}\right)+q_{2}(t)\right]=0, \quad t \in(0,1), \\
x^{(j)}(0)=y^{(j)}(0)=0, j=0,1,2, \cdots, n-2, \\
D_{0^{+}}^{\alpha-1} x(1)=\lambda_{1} \int_{0}^{\eta_{1}} y(t) d t \\
D_{0^{+}}^{\alpha-1} y(1)=\lambda_{2} \int_{0}^{\eta_{2}} x(t) d t
\end{array}\right.
$$

where

$$
\left[x(t)-\omega_{1}(t)\right]^{*}= \begin{cases}x(t)-\omega_{1}(t), & x(t)-\omega_{1}(t) \geqslant 0 \\ 0, & x(t)-\omega_{1}(t)<0\end{cases}
$$




$$
\left[y(t)-\omega_{2}(t)\right]^{*}= \begin{cases}y(t)-\omega_{2}(t), & y(t)-\omega_{2}(t) \geqslant 0 \\ 0, & y(t)-\omega_{2}(t)<0\end{cases}
$$

and

$$
\omega_{i}(t)=\lambda \int_{0}^{1} G_{i}(t, s) q_{i}(s) d s+\lambda \int_{0}^{1} H_{i}(t, s) q_{j}(s) d s, i=1,2, i+j=3,
$$

which is the solution of the fractional BVP

$$
\left\{\begin{array}{l}
D_{0^{+}}^{\alpha} \omega_{i}(t)+\lambda q_{i}(t)=0, \quad t \in(0,1), i=1,2, \\
\omega_{1}^{(j)}(0)=\omega_{2}^{(j)}(0)=0, j=0,1,2, \cdots, n-2, \\
D_{0^{+}}^{\alpha-1} \omega_{1}(1)=\lambda_{1} \int_{0}^{\eta_{1}} \omega_{2}(t) d t \\
D_{0^{+}}^{\alpha-1} \omega_{2}(1)=\lambda_{2} \int_{0}^{\eta_{2}} \omega_{1}(t) d t .
\end{array}\right.
$$

Obviously, by Lemma 2.6, one has

$$
\omega_{i}(t) \leqslant \lambda \rho t^{\alpha-1} \int_{0}^{1}\left(q_{1}(s)+q_{2}(s)\right) d s, i=1,2 .
$$

We shall prove that there exists a solution $(x, y)$ for fractional BVP (2.11) with $x(t) \geqslant \omega_{1}(t)$ and $y(t) \geqslant \omega_{2}(t)$ for all $t \in[0,1]$. If this is true, then the functions $u(t)=x(t)-w_{1}(t)$ and $v(t)=y(t)-w_{2}(t)$ represent a nonnegative solution of the SBVP (1.1), which is positive on $(0,1]$. Indeed, by the definition of $[\cdot]^{*}$, for any $t \in[0,1]$, we have

$$
\begin{aligned}
& D_{0^{+}}^{\alpha} u(t)=D_{0^{+}}^{\alpha} x(t)-D_{0^{+}}^{\alpha} \omega_{1}(t)=-\lambda f\left(t, x(t)-\omega_{1}(t), y(t)-\omega_{2}(t)\right)=-\lambda f(t, u(t), v(t)), \\
& D_{0^{+}}^{\alpha} v(t)=D_{0^{+}}^{\alpha} y(t)-D_{0^{+}}^{\alpha} \omega_{2}(t)=-\lambda g\left(t, x(t)-\omega_{1}(t), y(t)-\omega_{2}(t)\right)=-\lambda g(t, u(t), v(t)),
\end{aligned}
$$

and

$$
\begin{aligned}
u^{(j)}(0) & =x^{(j)}(0)-\omega_{1}^{(j)}(0)=0, v^{(j)}(0)=y^{(j)}(0)-\omega_{2}^{(j)}(0)=0, j=0,1,2, \cdots, n-2, \\
D_{0^{+}}^{\alpha} u(1) & =D_{0^{+}}^{\alpha} x(1)-D_{0^{+}}^{\alpha} \omega_{1}(1)=\lambda_{1} \int_{0}^{\eta_{1}}\left(y(t)-\omega_{2}(t)\right) d t=\lambda_{1} \int_{0}^{\eta_{1}} v(t) d t, \\
D_{0^{+}}^{\alpha} v(1) & =D_{0^{+}}^{\alpha} y(1)-D_{0^{+}}^{\alpha} \omega_{2}(1)=\lambda_{2} \int_{0}^{\eta_{2}}\left(x(t)-\omega_{1}(t)\right) d t=\lambda_{2} \int_{0}^{\eta_{2}} u(t) d t .
\end{aligned}
$$

Consequently, in what follows, we shall investigate BVP (2.11).

By means of Lemma 2.3, BVP (2.11) can be reduced to the following equivalent integral equation

$$
\left\{\begin{aligned}
x(t)= & \lambda \int_{0}^{1} G_{1}(t, s)\left[f\left(s,\left[x(s)-\omega_{1}(s)\right]^{*},\left[y(s)-\omega_{2}(s)\right]^{*}\right)+q_{1}(s)\right] d s \\
& +\lambda \int_{0}^{1} H_{1}(t, s)\left[g\left(s,\left[x(s)-\omega_{1}(s)\right]^{*},\left[y(s)-\omega_{2}(s)\right]^{*}\right)+q_{2}(s)\right] d s, t \in[0,1] \\
y(t)= & \lambda \int_{0}^{1} G_{2}(t, s)\left[g\left(s,\left[x(s)-\omega_{1}(s)\right]^{*},\left[y(s)-\omega_{2}(s)\right]^{*}\right)+q_{2}(s)\right] d s \\
& +\lambda \int_{0}^{1} H_{2}(t, s)\left[f\left(s,\left[x(s)-\omega_{1}(s)\right]^{*},\left[y(s)-\omega_{2}(s)\right]^{*}\right)+q_{1}(s)\right] d s, t \in[0,1] .
\end{aligned}\right.
$$

Define an integral operator $\mathrm{T}: \mathrm{P} \rightarrow \mathrm{E} \times \mathrm{E}$ by

$$
T(x, y)=\left(T_{1}(x, y), T_{2}(x, y)\right)
$$


with

$$
\begin{aligned}
& \mathrm{T}_{1}(x, y)(t)=\lambda \int_{0}^{1} G_{1}(t, s)\left[f\left(s,\left[x(s)-\omega_{1}(s)\right]^{*},\left[y(s)-\omega_{2}(s)\right]^{*}\right)+q_{1}(s)\right] d s \\
& +\lambda \int_{0}^{1} H_{1}(t, s)\left[g\left(s,\left[x(s)-\omega_{1}(s)\right]^{*},\left[y(s)-\omega_{2}(s)\right]^{*}\right)+q_{2}(s)\right] d s, \quad t \in[0,1], \\
& T_{2}(x, y)(t)=\lambda \int_{0}^{1} G_{2}(t, s)\left[g\left(s,\left[x(s)-\omega_{1}(s)\right]^{*},\left[y(s)-\omega_{2}(s)\right]^{*}\right)+q_{2}(s)\right] d s \\
& +\lambda \int_{0}^{1} H_{2}(t, s)\left[f\left(s,\left[x(s)-\omega_{1}(s)\right]^{*},\left[y(s)-\omega_{2}(s)\right]^{*}\right)+q_{1}(s)\right] d s, t \in[0,1] .
\end{aligned}
$$

Lemma 2.7. Suppose $(\mathrm{H} 1)$ or $\left(\mathrm{H} 1^{*}\right)$ holds, then the operator $\mathrm{T}: \mathrm{P} \rightarrow \mathrm{P}$ is a completely continuous operator.

Proof. For any fixed $(x, y) \in P$, we can choose a constant $C$ such that

$$
\begin{aligned}
& 0 \leqslant\left[x(s)-\omega_{1}(s)\right]^{*} \leqslant x(s) \leqslant\|(x, y)\|_{1} \leqslant C, s \in[0,1], \\
& 0 \leqslant\left[y(s)-\omega_{2}(s)\right]^{*} \leqslant y(s) \leqslant\|(x, y)\|_{1} \leqslant C, s \in[0,1] .
\end{aligned}
$$

Take $M_{i}=\max _{0 \leqslant u, v \leqslant C} h_{i}(u, v)+1, i=1$, 2. For any $t \in[0,1]$, the condition (H1) or (H1*) and Lemma 2.6 imply that

$$
\begin{aligned}
\mathrm{T}_{1}(x, y)(\mathrm{t}) \leqslant & \lambda \rho \int_{0}^{1}\left[1-(1-s)^{\alpha-1}\right]\left[\mathrm{f}\left(\mathrm{s},\left[x(s)-\omega_{1}(s)\right]^{*},\left[y(s)-\omega_{2}(s)\right]^{*}\right)+q_{1}(s)\right] d s \\
& +\lambda \rho \int_{0}^{1}\left[1-(1-s)^{\alpha-1}\right]\left[g\left(s,\left[x(s)-\omega_{1}(s)\right]^{*},\left[y(s)-\omega_{2}(s)\right]^{*}\right)+q_{2}(s)\right] d s \\
\leqslant & \lambda \rho \int_{0}^{1} p_{1}(s) h_{1}\left(\left[x(s)-\omega_{1}(s)\right]^{*},\left[y(s)-\omega_{2}(s)\right]^{*}\right) d s \\
& +\lambda \rho \int_{0}^{1} p_{2}(s) h_{2}\left(\left[x(s)-\omega_{1}(s)\right]^{*},\left[y(s)-\omega_{2}(s)\right]^{*}\right) d s \\
\leqslant & \lambda \rho M_{1} \int_{0}^{1} p_{1}(s) d s+\lambda \rho M_{2} \int_{0}^{1} p_{2}(s) d s<+\infty, \\
\mathrm{T}_{2}(x, y)(t) \leqslant & \lambda \rho \int_{0}^{1}\left[1-(1-s)^{\alpha-1}\right]\left[g\left(s,\left[x(s)-\omega_{1}(s)\right]^{*},\left[y(s)-\omega_{2}(s)\right]^{*}\right)+q_{2}(s)\right] d s \\
& +\lambda \rho \int_{0}^{1}\left[1-(1-s)^{\alpha-1}\right]\left[f\left(s,\left[x(s)-\omega_{1}(s)\right]^{*},\left[y(s)-\omega_{2}(s)\right]^{*}\right)+q_{1}(s)\right] d s \\
\leqslant & \lambda \rho \int_{0}^{1} p_{2}(s) h_{2}\left(\left[x(s)-\omega_{1}(s)\right]^{*},\left[y(s)-\omega_{2}(s)\right]^{*}\right) d s \\
& +\lambda \rho \int_{0}^{1} p_{1}(s) h_{1}\left(\left[x(s)-\omega_{1}(s)\right]^{*},\left[y(s)-\omega_{2}(s)\right]^{*}\right) d s \\
\leqslant & \lambda \rho M_{2} \int_{0}^{1} p_{2}(s) d s+\lambda \rho M_{1} \int_{0}^{1} p_{1}(s) d s<+\infty,
\end{aligned}
$$

which yields that the operator $\mathrm{T}$ is well-defined on $\mathrm{P}$.

On the other hand, for any fixed $(x, y) \in P, t \in[0,1]$, by Lemma 2.6, it follows that

$$
\mathrm{T}_{1}(x, y)(t) \leqslant \lambda \rho \int_{0}^{1}\left[1-(1-s)^{\alpha-1}\right]\left[f\left(s,\left[x(s)-\omega_{1}(s)\right]^{*},\left[y(s)-\omega_{2}(s)\right]^{*}\right)+q_{1}(s)\right] d s
$$




$$
\begin{aligned}
& +\lambda \rho \int_{0}^{1}\left[1-(1-s)^{\alpha-1}\right]\left[g\left(s,\left[x(s)-\omega_{1}(s)\right]^{*},\left[y(s)-\omega_{2}(s)\right]^{*}\right)+q_{2}(s)\right] d s \\
\mathrm{~T}_{2}(x, y)(t) \leqslant & \lambda \rho \int_{0}^{1}\left[1-(1-s)^{\alpha-1}\right]\left[g\left(s,\left[x(s)-\omega_{1}(s)\right]^{*},\left[y(s)-\omega_{2}(s)\right]^{*}\right)+q_{2}(s)\right] d s \\
& +\lambda \rho \int_{0}^{1}\left[1-(1-s)^{\alpha-1}\right]\left[f\left(s,\left[x(s)-\omega_{1}(s)\right]^{*},\left[y(s)-\omega_{2}(s)\right]^{*}\right)+q_{1}(s)\right] d s
\end{aligned}
$$

which shows that

$$
\begin{aligned}
\left\|\mathrm{T}_{1}(x, y)\right\| \leqslant & \lambda \rho \int_{0}^{1}\left[1-(1-s)^{\alpha-1}\right]\left[f\left(s,\left[x(s)-\omega_{1}(s)\right]^{*},\left[y(s)-\omega_{2}(s)\right]^{*}\right)+q_{1}(s)\right] d s \\
& +\lambda \rho \int_{0}^{1}\left[1-(1-s)^{\alpha-1}\right]\left[g\left(s,\left[x(s)-\omega_{1}(s)\right]^{*},\left[y(s)-\omega_{2}(s)\right]^{*}\right)+q_{2}(s)\right] d s \\
\left\|T_{2}(x, y)\right\| \leqslant & \lambda \rho \int_{0}^{1}\left[1-(1-s)^{\alpha-1}\right]\left[g\left(s,\left[x(s)-\omega_{1}(s)\right]^{*},\left[y(s)-\omega_{2}(s)\right]^{*}\right)+q_{2}(s)\right] d s \\
& +\lambda \rho \int_{0}^{1}\left[1-(1-s)^{\alpha-1}\right]\left[f\left(s,\left[x(s)-\omega_{1}(s)\right]^{*},\left[y(s)-\omega_{2}(s)\right]^{*}\right)+q_{1}(s)\right] d s .
\end{aligned}
$$

From Lemma 2.6, we have

$$
\begin{aligned}
& \mathrm{T}_{1}(x, y)(\mathrm{t}) \geqslant \lambda v \mathrm{t}^{\alpha-1} \int_{0}^{1}\left[1-(1-s)^{\alpha-1}\right]\left[f\left(s,\left[x(s)-\omega_{1}(s)\right]^{*},\left[y(s)-\omega_{2}(s)\right]^{*}\right)+q_{1}(s)\right] d s \\
& +\lambda v t^{\alpha-1} \int_{0}^{1}\left[1-(1-s)^{\alpha-1}\right]\left[g\left(s,\left[x(s)-\omega_{1}(s)\right]^{*},\left[y(s)-\omega_{2}(s)\right]^{*}\right)+q_{2}(s)\right] d s, \\
& \mathrm{~T}_{2}(x, y)(t) \geqslant \lambda v t^{\alpha-1} \int_{0}^{1}\left[1-(1-s)^{\alpha-1}\right]\left[g\left(s,\left[x(s)-\omega_{1}(s)\right]^{*},\left[y(s)-\omega_{2}(s)\right]^{*}\right)+q_{2}(s)\right] d s \\
& +\lambda v t^{\alpha-1} \int_{0}^{1}\left[1-(1-s)^{\alpha-1}\right]\left[f\left(s,\left[x(s)-\omega_{1}(s)\right]^{*},\left[y(s)-\omega_{2}(s)\right]^{*}\right)+q_{1}(s)\right] d s .
\end{aligned}
$$

Then, (2.13), (2.14), (2.15), and (2.16) imply that

$$
\mathrm{T}_{1}(x, y)(\mathrm{t}) \geqslant \gamma \mathrm{t}^{\alpha-1}\left\|\mathrm{~T}_{1}(x, y)\right\|_{1}, \mathrm{~T}_{2}(x, y)(\mathrm{t}) \geqslant \gamma \mathrm{t}^{\alpha-1}\left\|\mathrm{~T}_{2}(x, y)(\mathrm{t})\right\|_{1}, \mathrm{t} \in[0,1],
$$

which means $\mathrm{T}(\mathrm{P}) \subset \mathrm{P}$.

By means of Arzela-Ascoli theorem, it is easy to see that $\mathrm{T}: \mathrm{P} \rightarrow \mathrm{P}$ is a completely continuous operator.

For convenience, we also list the following lemmas which will be used in the next section.

Lemma 2.8 ([2]). Let $\mathrm{X}$ be a Banach space, and $\Omega \subset \mathrm{X}$ be closed and convex. Assume that $\mathrm{U}$ is a relatively open subsets of $\Omega$ with $0 \in \mathrm{U}$.S : $\overline{\mathrm{U}} \rightarrow \Omega$ is a compact, continuous map. Then either

(i) S has a fixed point in $\overline{\mathrm{U}}$; or

(ii) there exist $u \in \partial U$ and $v \in(0,1)$ such that $u=v S u$.

Lemma 2.9 ([8]). Let $\mathrm{E}$ be a Banach space, $\mathrm{P} \subseteq \mathrm{E}$ a cone, and $\Omega_{1}, \Omega_{2}$ two bounded open balls of $\mathrm{E}$ centered at the origin with $\bar{\Omega}_{1} \subset \Omega_{2}$. Suppose that $\mathrm{T}: \mathrm{P} \cap\left(\bar{\Omega}_{2} \backslash \Omega_{1}\right) \rightarrow \mathrm{P}$ is a completely continuous operator such that either

(i) $\|\mathrm{T} x\| \leqslant\|x\|, x \in \mathrm{P} \cap \partial \Omega_{1}$ and $\|\mathrm{T} x\| \geqslant\|x\|, x \in \mathrm{P} \cap \partial \Omega_{2} ;$ or

(ii) $\|\mathrm{T} x\| \geqslant\|x\|, x \in P \cap \partial \Omega_{1}$ and $\|\mathrm{T} x\| \leqslant\|x\|, x \in P \cap \partial \Omega_{2}$

holds. Then $\mathrm{T}$ has a fixed point in $\mathrm{P} \cap\left(\bar{\Omega}_{2} \backslash \Omega_{1}\right)$.

Lemma 2.10 ([8]). Let $\mathrm{E}$ be a Banach space, $\mathrm{P}$ be a cone in $\mathrm{E}$ and $\Omega \in \mathrm{E}$ is a bounded open set with $\theta \in \Omega$. Suppose that $\mathrm{A}: \bar{\Omega} \cap \mathrm{P} \rightarrow \mathrm{P}$ is completely continuous. Then the following results hold:

(i) If $u \neq \mu A u$ for $\mu \in \partial \Omega \cap P$ and $0 \leqslant \mu \leqslant 1$, then $i(A, \Omega \cap P, P)=1$.

(ii) If $\inf _{\mathfrak{u} \in \mathrm{P} \cap \partial \Omega}\|A u\|>0$ and $A u \neq \mu u$ for any $u \in \partial \Omega \cap P$ and $0<\mu \leqslant 1$, then $i(A, \Omega \cap P, P)=0$. 


\section{Main results}

The following two theorems are our main results which give sufficient conditions to guarantee the existence of at least two positive solutions for system (1.1).

Theorem 3.1. Suppose that conditions (H1), (H2), and (H3) hold. Then there exists a constant $\lambda^{*}>0$ such that $S B V P(1.1)$ has at least two positive solutions for any $\lambda \in\left(0, \lambda^{*}\right)$.

Proof. From (H2), there exists a constant $\mathrm{R}_{0}>0$ such that

$$
f(t, u, v)>0, g(t, u, v)>0, \forall u, v \in\left[0, R_{0}\right]
$$

Define

$$
F\left(R_{0}\right)=\max _{\substack{0 \leqslant t \leqslant 1 \\ 0 \leqslant u, v \leqslant R_{0}}}\{f(t, u, v)\}, \quad G\left(R_{0}\right)=\max _{\substack{0 \leqslant t \leqslant 1 \\ 0 \leqslant u, v \leqslant R_{0}}}\{g(t, u, v)\}
$$

and

$$
\bar{\lambda}=\frac{R_{0}}{\rho\left(\left(F\left(R_{0}\right)+G\left(R_{0}\right)\right)\left(1-\frac{1}{\alpha}\right)+\int_{0}^{1}\left(1-(1-s)^{\alpha-1}\right)\left(q_{1}(s)+q_{2}(s)\right) d s\right)}>0 .
$$

Let $\mathrm{U}=\left\{(x, y) \in P:\|(x, y)\|_{1}<R_{0}\right\}$. Assume that there exist $(x, y) \in \partial U$ and $v \in(0,1)$ such that $(x, y)=v \top(x, y)$. Notice that

$$
0 \leqslant\left[x(s)-\omega_{1}(s)\right]^{*} \leqslant x(s) \leqslant\|(x, y)\|_{1}=R_{0}, \quad 0 \leqslant\left[y(s)-\omega_{2}(s)\right]^{*} \leqslant y(s) \leqslant\|(x, y)\|_{1}=R_{0} .
$$

Then, for each $\lambda \in(0, \bar{\lambda})$, by Lemma 2.6 , we have

$$
\begin{aligned}
x(t)=v T_{1}(x, y)(t) \leqslant & T_{1}(x, y)(t) \\
= & \lambda \int_{0}^{1} G_{1}(t, s)\left[f\left(s,\left[x(s)-\omega_{1}(s)\right]^{*},\left[y(s)-\omega_{2}(s)\right]^{*}\right)+q_{1}(s)\right] d s \\
& +\lambda \int_{0}^{1} H_{1}(t, s)\left[g\left(s,\left[x(s)-\omega_{1}(s)\right]^{*},\left[y(s)-\omega_{2}(s)\right]^{*}\right)+q_{2}(s)\right] d s \\
\leqslant & \lambda \rho \int_{0}^{1}\left(1-(1-s)^{\alpha-1}\right)\left(F\left(R_{0}\right)+q_{1}(s)\right) d s+\lambda \rho \int_{0}^{1}\left(1-(1-s)^{\alpha-1}\right)\left(G\left(R_{0}\right)+q_{2}(s)\right) d s \\
\leqslant & \lambda \rho\left(\left(F\left(R_{0}\right)+G\left(R_{0}\right)\right)\left(1-\frac{1}{\alpha}\right)+\int_{0}^{1}\left(1-(1-s)^{\alpha-1}\right)\left(q_{1}(s)+q_{2}(s)\right) d s\right) \\
< & R_{0}, \forall t \in[0,1], \\
y(t)=v T_{2}(x, y)(t) \leqslant & T_{2}(x, y)(t) \\
= & \lambda \int_{0}^{1} G_{2}(t, s)\left[g\left(s,\left[x(s)-\omega_{1}(s)\right]^{*},\left[y(s)-\omega_{2}(s)\right]^{*}\right)+q_{2}(s)\right] d s \\
& +\lambda \int_{0}^{1} H_{2}(t, s)\left[f\left(s,\left[x(s)-\omega_{1}(s)\right]^{*},\left[y(s)-\omega_{2}(s)\right]^{*}\right)+q_{1}(s)\right] d s \\
\leqslant & \lambda \rho \int_{0}^{1}\left(1-(1-s)^{\alpha-1}\right)\left(G\left(R_{0}\right)+q_{2}(s)\right) d s+\lambda \rho \int_{0}^{1}\left(1-(1-s)^{\alpha-1}\right)\left(F\left(R_{0}\right)+q_{1}(s)\right) d s \\
\leqslant & \lambda \rho\left(\left(F\left(R_{0}\right)+G\left(R_{0}\right)\right)\left(1-\frac{1}{\alpha}\right)+\int_{0}^{1}\left(1-(1-s)^{\alpha-1}\right)\left(q_{1}(s)+q_{2}(s)\right) d s\right) \\
< & R_{0}, \forall t \in[0,1],
\end{aligned}
$$

which means $\|x\|<R_{0}$ and $\|y\|<R_{0}$. Thus, $R_{0}=\|(x, y)\|_{1}=\max \{\|x\|,\|y\|\}<R_{0}$. This is a contradiction. By Lemma 2.8, T has a fixed point $\left(x_{1}, y_{1}\right) \in \overline{\mathrm{U}}$. 
Moreover, by (3.1), it follows that

$$
\begin{aligned}
x_{1}(t)= & T_{1}\left(x_{1}, y_{1}\right)(t) \\
= & \lambda \int_{0}^{1} G_{1}(t, s)\left[f\left(s,\left[x_{1}(s)-\omega_{1}(s)\right]^{*},\left[y_{1}(s)-\omega_{2}(s)\right]^{*}\right)+q_{1}(s)\right] d s \\
& +\lambda \int_{0}^{1} H_{1}(t, s)\left[g\left(s,\left[x_{1}(s)-\omega_{1}(s)\right]^{*},\left[y_{1}(s)-\omega_{2}(s)\right]^{*}\right)+q_{2}(s)\right] d s \\
> & \lambda \int_{0}^{1} G_{1}(t, s) q_{1}(s) d s+\lambda \int_{0}^{1} H_{1}(t, s) q_{2}(s) d s \\
= & \omega_{1}(t), \forall t \in(0,1], \\
y_{1}(t)= & T_{2}\left(x_{1}, y_{1}\right)(t) \\
= & \lambda \int_{0}^{1} G_{2}(t, s)\left[g\left(s,\left[x_{1}(s)-\omega_{1}(s)\right]^{*},\left[y_{1}(s)-\omega_{2}(s)\right]^{*}\right)+q_{2}(s)\right] d s \\
& +\lambda \int_{0}^{1} H_{2}(t, s)\left[f\left(s,\left[x_{1}(s)-\omega_{1}(s)\right]^{*},\left[y_{1}(s)-\omega_{2}(s)\right]^{*}\right)+q_{1}(s)\right] d s \\
> & \lambda \int_{0}^{1} G_{2}(t, s) q_{2}(s) d s+\lambda \int_{0}^{1} H_{2}(t, s) q_{1}(s) d s \\
= & \omega_{2}(t), \forall t \in(0,1] .
\end{aligned}
$$

Thus, $x_{1}(t)-\omega_{1}(t)>0$ and $y_{1}(t)-\omega_{2}(t)>0$ for all $t \in(0,1]$.

Let $u_{1}(t)=x_{1}(t)-w_{1}(t)$ and $v_{1}(t)=y_{1}(t)-w_{2}(t)$. Then $\left(u_{1}, v_{1}\right)$ is a nonnegative solution (positive on $(0,1])$ of the SBVP $(1.1)$.

On the other hand, choose $R_{1}$ such that $R_{1}>\max \left\{\frac{\rho}{\gamma^{2}} \int_{0}^{1}\left(q_{1}(s)+q_{2}(s)\right) d s, R_{0}\right\}$. Define

$$
F\left(R_{1}\right)=\max _{\substack{0 \leqslant t \leqslant 1 \\ 0 \leqslant u, v \leqslant R_{1}}}\{f(t, u, v)\}, \quad G\left(R_{1}\right)=\max _{\substack{0 \leqslant t \leqslant 1 \\ 0 \leqslant u, v \leqslant R_{1}}}\{g(t, u, v)\},
$$

and

$$
\widetilde{\lambda}=\min \left\{1, \frac{R_{1}}{\rho\left(\left(F\left(R_{1}\right)+G\left(R_{1}\right)\right)\left(1-\frac{1}{\alpha}\right)+\int_{0}^{1}\left(1-(1-s)^{\alpha-1}\right)\left(q_{1}(s)+q_{2}(s)\right) d s\right)}\right\}>0 .
$$

Let $\Omega_{1}=\left\{(x, y) \in E:\|(x, y)\|_{1}<R_{1}\right\}$. Then, for any $(x, y) \in P \cap \partial \Omega_{1}, s \in[0,1]$, we have

$$
0 \leqslant\left[x(s)-\omega_{1}(s)\right]^{*} \leqslant x(s) \leqslant\|(x, y)\|_{1}=R_{1}, \quad 0 \leqslant\left[y(s)-\omega_{2}(s)\right]^{*} \leqslant y(s) \leqslant\|(x, y)\|_{1}=R_{1} .
$$

For each $\lambda \in(0, \tilde{\lambda})$, it follows from Lemma 2.6 that

$$
\begin{aligned}
\left\|\mathrm{T}_{1}(x, y)\right\| \leqslant & \lambda \rho \int_{0}^{1}\left(1-(1-s)^{\alpha-1}\right)\left(f\left(s,\left[x(s)-\omega_{1}(s)\right]^{*},\left[y(s)-\omega_{2}(s)\right]^{*}\right)+q_{1}(s)\right) d s \\
& +\lambda \rho \int_{0}^{1}\left(1-(1-s)^{\alpha-1}\right)\left[g\left(s,\left[x(s)-\omega_{1}(s)\right]^{*},\left[y(s)-\omega_{2}(s)\right]^{*}\right)+q_{2}(s)\right] d s \\
\leqslant & \lambda \rho \int_{0}^{1}\left(1-(1-s)^{\alpha-1}\right)\left(F\left(R_{1}\right)+q_{1}(s)\right) d s+\lambda \rho \int_{0}^{1}\left(1-(1-s)^{\alpha-1}\right)\left(G\left(R_{1}\right)+q_{2}(s)\right) d s \\
\leqslant & \lambda \rho\left(\left(F\left(R_{1}\right)+G\left(R_{1}\right)\right)\left(1-\frac{1}{\alpha}\right)+\int_{0}^{1}\left(1-(1-s)^{\alpha-1}\right)\left(q_{1}(s)+q_{2}(s)\right) d s\right) \\
\leqslant & \|(x, y)\|_{1}=R_{1},
\end{aligned}
$$




$$
\begin{aligned}
\left\|\mathrm{T}_{2}(x, y)\right\|= & \lambda \rho \int_{0}^{1}\left(1-(1-s)^{\alpha-1}\right)\left[g\left(s,\left[x(s)-\omega_{1}(s)\right]^{*},\left[y(s)-\omega_{2}(s)\right]^{*}\right)+q_{2}(s)\right] d s \\
& +\lambda \rho \int_{0}^{1}\left(1-(1-s)^{\alpha-1}\right)\left[f\left(s,\left[x(s)-\omega_{1}(s)\right]^{*},\left[y(s)-\omega_{2}(s)\right]^{*}\right)+q_{1}(s)\right] d s \\
\leqslant & \lambda \rho \int_{0}^{1}\left(1-(1-s)^{\alpha-1}\right)\left(G\left(R_{1}\right)+q_{2}(s)\right) d s+\lambda \rho \int_{0}^{1}\left(1-(1-s)^{\alpha-1}\right)\left(F\left(R_{1}\right)+q_{1}(s)\right) d s \\
\leqslant & \lambda \rho\left(\left(F\left(R_{1}\right)+G\left(R_{1}\right)\right)\left(1-\frac{1}{\alpha}\right)+\int_{0}^{1}\left(1-(1-s)^{\alpha-1}\right)\left(q_{1}(s)+q_{2}(s)\right) d s\right) \\
\leqslant & \|(x, y)\|_{1}=R_{1} .
\end{aligned}
$$

Therefore,

$$
\|\mathrm{T}(x, y)\|_{1}=\max \left\{\left\|\mathrm{T}_{1}(x, y)\right\|,\left\|\mathrm{T}_{2}(x, y)\right\|\right\} \leqslant\|(x, y)\|_{1}, \forall(x, y) \in \mathrm{P} \cap \partial \Omega_{1} .
$$

By (H3), if $f_{\infty}=+\infty$, then there exists a constant $\mathrm{N}$ for $\mathrm{f}$ such that

$$
f(t, u, v) \geqslant M(u+v), t \in[\xi, \eta], u \geqslant 0, v \geqslant 0, u+v \geqslant N,
$$

where $M$ satisfies

$$
\frac{1}{2} \lambda v \xi^{2(\alpha-1)} \gamma M \int_{\xi}^{\eta}\left(1-(1-s)^{\alpha-1}\right) \mathrm{d} s \geqslant 1
$$

Let $R_{2}=\max \left\{2 R_{1}, \frac{2 N}{\gamma \xi^{\alpha-1}}\right\}$ and $\Omega_{2}=\left\{(x, y) \in E:\|(x, y)\|_{1}<R_{2}\right\}$. Then, by (2.12), for any $(x, y) \in$ $\mathrm{P} \cap \partial \Omega_{1}, \mathrm{~s} \in[0,1]$, we have

$$
\begin{aligned}
x(t)-\omega_{1}(t) & \geqslant x(t)-\lambda \rho t^{\alpha-1} \int_{0}^{1}\left(q_{1}(s)+q_{2}(s)\right) d s \\
& \geqslant x(t)-\frac{\rho x(t)}{\gamma\|x\|} \int_{0}^{1}\left(q_{1}(s)+q_{2}(s)\right) d s \\
& \geqslant x(t)-\frac{\rho x(t)}{\gamma R_{2}} \int_{0}^{1}\left(q_{1}(s)+q_{2}(s)\right) d s \\
& \geqslant \frac{1}{2} x(t) \geqslant \frac{1}{2} \gamma t^{\alpha-1}\|(x, y)\|_{1} \geqslant \frac{1}{2} \gamma \xi^{\alpha-1} R_{2} \geqslant N
\end{aligned}
$$

and then

$$
\left[x(t)-\omega_{1}(t)\right]^{*}+\left[y(t)-\omega_{2}(t)\right]^{*} \geqslant\left[x(t)-\omega_{1}(t)\right]^{*}=x(t)-\omega_{1}(t) \geqslant N .
$$

Hence, for any $(x, y) \in P \cap \partial \Omega_{2}, t \in[\xi, \eta]$, by (3.3) and (3.5), we get

$$
f\left(t,\left[x(t)-\omega_{1}(t)\right]^{*},\left[y(t)-\omega_{2}(t)\right]^{*}\right) \geqslant M\left(\left[x(t)-\omega_{1}(t)\right]^{*}+\left[y(t)-\omega_{2}(t)\right]^{*}\right) .
$$

Therefore, for any $(x, y) \in P \cap \partial \Omega_{2}, t \in[\xi, \eta]$, by (3.4), (3.5), and (3.6), we have

$$
\begin{aligned}
\mathrm{T}_{1}(x, y)(t) & \geqslant \lambda \int_{0}^{1} \mathrm{G}_{1}(\mathrm{t}, \mathrm{s})\left[\mathrm{f}\left(\mathrm{s},\left[\mathrm{x}(\mathrm{s})-\omega_{1}(\mathrm{~s})\right]^{*},\left[\mathrm{y}(\mathrm{s})-\omega_{2}(\mathrm{~s})\right]^{*}\right)+\mathrm{q}_{1}(\mathrm{~s})\right] \mathrm{d} s \\
& \geqslant \lambda v \mathrm{t}^{\alpha-1} \int_{0}^{1}\left(1-(1-s)^{\alpha-1}\right) f\left(s,\left[x(s)-\omega_{1}(s)\right]^{*},\left[y(s)-\omega_{2}(s)\right]^{*}\right) d s \\
& \geqslant \lambda v t^{\alpha-1} M\left(\left[x(s)-\omega_{1}(s)\right]^{*}+\left[y(s)-\omega_{2}(s)\right]^{*}\right) \int_{\xi}^{\eta}\left(1-(1-s)^{\alpha-1}\right) d s \\
& \geqslant \frac{1}{2} \lambda v \xi^{2(\alpha-1)} \gamma M R_{2} \int_{\xi}^{\eta}\left(1-(1-s)^{\alpha-1}\right) d s
\end{aligned}
$$




$$
\geqslant R_{2}=\|(x, y)\|_{1}
$$

which implies that

$$
\|\mathrm{T}(x, y)\|_{1}=\max \left\{\left\|\mathrm{T}_{1}(x, y)\right\|,\left\|\mathrm{T}_{2}(x, y)\right\|\right\} \geqslant\|(x, y)\|_{1}, \forall(x, y) \in \mathrm{P} \cap \partial \Omega_{2} .
$$

Similarly (3.7) is valid if $g_{\infty}=+\infty$.

It follows from (3.2) and (3.7) that $T$ has a fixed point $\left(x_{2}, y_{2}\right) \in P$ which satisfies $R_{1} \leqslant\left\|\left(x_{2}, y_{2}\right)\right\|_{1} \leqslant R_{2}$. We suppose that $\left\|x_{2}\right\| \geqslant R_{1}$ without loss of generality. Then, by (2.12) and the choice of $R_{1}$, we have

$$
\begin{aligned}
x_{2}(t)-\omega_{1}(t) & \geqslant x_{2}(t)-\lambda \rho t^{\alpha-1} \int_{0}^{1}\left(q_{1}(s)+q_{2}(s)\right) d s \\
& \geqslant x_{2}(t)-\frac{\rho x_{2}(t)}{\gamma\left\|x_{2}\right\|} \int_{0}^{1}\left(q_{1}(s)+q_{2}(s)\right) d s \\
& \geqslant\left(1-\frac{\rho}{\gamma R_{1}} \int_{0}^{1}\left(q_{1}(s)+q_{2}(s)\right) d s\right) x_{2}(t) \\
& \geqslant\left(1-\frac{\rho}{\gamma R_{1}} \int_{0}^{1}\left(q_{1}(s)+q_{2}(s)\right) d s\right) \gamma t^{\alpha-1}\left\|\left(x_{2}, y_{2}\right)\right\|_{1} \\
& \geqslant\left(1-\frac{\rho}{\gamma R_{1}} \int_{0}^{1}\left(q_{1}(s)+q_{2}(s)\right) d s\right) \gamma R_{1} t^{\alpha-1}>0, t \in(0,1] .
\end{aligned}
$$

Moreover, noticing $\left(x_{2}, y_{2}\right) \in P$, we can obtain $y_{2}(t) \geqslant \gamma t^{\alpha-1}\left\|\left(x_{2}, y_{2}\right)\right\|_{1} \geqslant \gamma t^{\alpha-1} R_{1}$ for $t \in[0,1]$, and then $\left\|y_{2}\right\| \geqslant y_{2}(1) \geqslant \gamma R_{1}$. Similarly, we also have

$$
\begin{aligned}
y_{2}(t)-\omega_{2}(t) & \geqslant y_{2}(t)-\lambda \rho t^{\alpha-1} \int_{0}^{1}\left(q_{1}(s)+q_{2}(s)\right) d s \\
& \geqslant y_{2}(t)-\frac{\rho y_{2}(t)}{\gamma\left\|y_{2}\right\|} \int_{0}^{1}\left(q_{1}(s)+q_{2}(s)\right) d s \\
& \geqslant\left(1-\frac{\rho}{\gamma^{2} R_{1}} \int_{0}^{1}\left(q_{1}(s)+q_{2}(s)\right) d s\right) y_{2}(t) \\
& \geqslant\left(1-\frac{\rho}{\gamma^{2} R_{1}} \int_{0}^{1}\left(q_{1}(s)+q_{2}(s)\right) d s\right) \gamma t^{\alpha-1}\left\|\left(x_{2}, y_{2}\right)\right\|_{1} \\
& \geqslant\left(1-\frac{\rho}{\gamma^{2} R_{1}} \int_{0}^{1}\left(q_{1}(s)+q_{2}(s)\right) d s\right) \gamma R_{1} t^{\alpha-1}>0, t \in(0,1]
\end{aligned}
$$

Let $u_{2}(t)=x_{2}(t)-w_{1}(t)$ and $v_{2}(t)=y_{2}(t)-\omega_{2}(t)$. Then, $\left(u_{2}, v_{2}\right)$ is a nonnegative solution (positive on $(0,1])$ of the SBVP $(1.1)$.

Choose $\lambda^{*}=\min \{\bar{\lambda}, \tilde{\lambda}\}$. Then for any $\lambda \in\left(0, \lambda^{*}\right)$, the SBVP (1.1) has at least two positive solutions $\left(u_{1}, v_{1}\right)$ and $\left(u_{2}, v_{2}\right)$.

Theorem 3.2. Suppose that conditions $\left(\mathrm{H}^{*}\right),(\mathrm{H} 3)$, and $(\mathrm{H} 4)$ hold. Then there exists a constant $\lambda^{*}>0$ such that $S B V P(1.1)$ has at least two positive solutions for any $\lambda \in\left(0, \lambda^{*}\right)$.

Proof. We divide the proof into 3 steps.

Step 1. For each $r>0$, let

$$
\bar{\lambda}=\min \left\{\frac{\gamma r}{2 \rho \int_{0}^{1}\left(q_{1}(s)+q_{2}(s)\right) d s}, \frac{r}{\rho e_{1}(r) \int_{0}^{1} p_{1}(s) d s+\rho e_{2}(r) \int_{0}^{1} p_{2}(s) d s}\right\},
$$


where

$$
e_{i}(r)=: \max _{(x, y) \in[0, r] \times[0, r]} h_{i}(x, y)+1
$$

For any $\lambda \in(0, \bar{\lambda})$, we shall prove

$$
(x, y) \neq \mu \mathrm{T}(x, y), \mu \in[0,1],(x, y) \in \partial \mathrm{P}_{\mathrm{r}} .
$$

In fact, if not, there exist $\left(x_{0}, y_{0}\right) \in \partial P_{r}$ and $\mu_{0} \in[0,1]$ such that

$$
\left(x_{0}, y_{0}\right)=\mu_{0} T\left(x_{0}, y_{0}\right), t \in[0,1]
$$

Since $\left(x_{0}, y_{0}\right) \in P$, one has

$$
x_{0}(t) \geqslant \gamma t^{\alpha-1}\left\|\left(x_{0}, y_{0}\right)\right\|_{1}=\gamma t^{\alpha-1} r, t \in[0,1] .
$$

On the other hand, from (2.12), we get

$$
\omega_{1}(t) \leqslant \lambda \rho t^{\alpha-1} \int_{0}^{1}\left(q_{1}(s)+q_{2}(s)\right) d s \leqslant \frac{\lambda \rho \int_{0}^{1}\left(q_{1}(s)+q_{2}(s)\right) d s}{\gamma r} x_{0}(t), t \in[0,1] .
$$

Thus,

$$
x_{0}(t)-\omega_{1}(t) \geqslant\left(1-\frac{\lambda \rho \int_{0}^{1}\left(q_{1}(s)+q_{2}(s)\right) d s}{\gamma r}\right) \geqslant \frac{1}{2} x_{0}(t) \geqslant \frac{\gamma r}{2} t^{\alpha-1}, t \in[0,1] .
$$

In addition,

$$
\left[x_{0}(t)-\omega_{1}(t)\right]^{*}=x_{0}(t)-\omega_{1}(t) \leqslant x_{0}(t) \leqslant\left\|\left(x_{0}, y_{0}\right)\right\|_{1}=r .
$$

By a similar way, we can obtain

$$
\frac{\gamma r}{2} t^{\alpha-1} \leqslant \frac{1}{2} y_{0}(t) \leqslant\left[y_{0}(t)-\omega_{2}(t)\right]^{*}=y_{0}(t)-\omega_{2}(t) \leqslant r, t \in[0,1] .
$$

Therefore, by (3.8), (3.9), (3.10), and $\left(\mathrm{H} 1^{*}\right)$, we have

$$
\begin{aligned}
x_{0}(t)=\mu_{0} T_{1}\left(x_{0}, y_{0}\right)(t) \leqslant & T_{1}\left(x_{0}, y_{0}\right)(t) \\
= & \lambda \int_{0}^{1} G_{1}(t, s)\left(f\left(s,\left[x_{0}(s)-\omega_{1}(s)\right]^{*},\left[y_{0}(s)-\omega_{2}(s)\right]^{*}\right)+q_{1}(s)\right) d s \\
& +\lambda \int_{0}^{1} H_{1}(t, s)\left(g\left(s,\left[x_{0}(s)-\omega_{1}(s)\right]^{*},\left[y_{0}(s)-\omega_{2}(s)\right]^{*}\right)+q_{2}(s)\right) d s \\
\leqslant & \lambda \rho \int_{0}^{1} p_{1}(s) h_{1}\left(x_{0}(s)-\omega_{1}(s), y_{0}(s)-\omega_{2}(s)\right) d s \\
& +\lambda \rho \int_{0}^{1} p_{2}(s) h_{2}\left(x_{0}(s)-\omega_{1}(s), y_{0}(s)-\omega_{2}(s)\right) d s \\
\leqslant & \lambda \rho e_{1}(r) \int_{0}^{1} p_{1}(s) d s+\lambda \rho e_{2}(r) \int_{0}^{1} p_{2}(s) d s, \quad t \in[0,1],
\end{aligned}
$$

which implies

$$
\left\|x_{0}\right\| \leqslant \lambda \rho e_{1}(r) \int_{0}^{1} p_{1}(s) d s+\lambda \rho e_{2}(r) \int_{0}^{1} p_{2}(s) d s .
$$

In the same way, we can obtain

$$
\left\|y_{0}\right\| \leqslant \lambda \rho e_{1}(r) \int_{0}^{1} p_{1}(s) d s+\lambda \rho e_{2}(r) \int_{0}^{1} p_{2}(s) d s .
$$


Then,

$$
r=\left\|\left(x_{0}, y_{0}\right)\right\|_{1}=\max \left\{\left\|x_{0}\right\|,\left\|y_{0}\right\|\right\} \leqslant \lambda \rho e_{1}(r) \int_{0}^{1} p_{1}(s) d s+\lambda \rho e_{2}(r) \int_{0}^{1} p_{2}(s) d s,
$$

which indicates

$$
\lambda \geqslant \frac{r}{\rho e_{1}(r) \int_{0}^{1} p_{1}(s) d s+\rho e_{2}(r) \int_{0}^{1} p_{2}(s) d s} .
$$

This is in contradiction with $\lambda \in(0, \bar{\lambda})$. By Lemma 2.10, for any $\lambda \in(0, \bar{\lambda})$, we have

$$
i\left(T, P_{r}, P\right)=1 \text {. }
$$

Step 2. We shall prove that for any $\lambda \in(0, \bar{\lambda})$, there exists $R>r>0$ such that $i\left(T, P_{R}, P\right)=0$. First, by (H3), if $f_{\infty}=+\infty$ then there exists a constant $N>r$ for $f$ such that

$$
f(t, u, v) \geqslant\left(\frac{1}{2} \lambda v \xi^{2(\alpha-1)} \gamma \int_{\xi}^{\eta}\left(1-(1-s)^{\alpha-1}\right) d s\right)^{-1}(u+v), t \in[\xi, \eta], u, v \geqslant 0, u+v \geqslant N .
$$

Next, let

$$
R>\max \left\{\frac{2 \lambda \rho}{\gamma} \int_{0}^{1}\left(q_{1}(s)+q_{2}(s)\right) d s, \frac{2 N}{\gamma \xi^{\alpha-1}}\right\}
$$

Now, we are ready to show

$$
\mathrm{T}(x, y) \neq \mu(x, y), \quad \forall(x, y) \in \partial P_{R}, \mu \in(0,1] .
$$

Otherwise, there exist $\left(x_{0}, y_{0}\right) \in \partial P_{R}$ and $\mu_{0} \in(0,1]$ such that $T\left(x_{0}, y_{0}\right)=\mu_{0}\left(x_{0}, y_{0}\right)$. Since $\left(x_{0}, y_{0}\right) \in P$, one has

$$
x_{0}(t) \geqslant \gamma t^{\alpha-1}\left\|\left(x_{0}, y_{0}\right)\right\|_{1}=\gamma t^{\alpha-1} R, \quad t \in[0,1] .
$$

This together with (2.12) gives

$$
x_{0}(t)-\omega_{1}(t) \geqslant t^{\alpha-1}\left(\gamma R-\lambda \rho \int_{0}^{1}\left(q_{1}(s)+q_{2}(s)\right) d s\right)>\frac{1}{2} \gamma \xi^{\alpha-1} R>N, t \in[\xi, \eta] .
$$

Similarly, $y_{0}(t)-\omega_{2}(t)>N$ for any $t \in[\xi, \eta]$, and then

$$
\left[x_{0}(t)-\omega_{1}(t)\right]^{*}+\left[y_{0}(t)-\omega_{2}(t)\right]^{*} \geqslant\left[x_{0}(t)-\omega_{1}(t)\right]^{*}=x_{0}(t)-\omega_{1}(t)>\frac{1}{2} \gamma \xi^{\alpha-1} R>N .
$$

Hence, by (3.12) and (3.13), we have

$$
\begin{aligned}
x_{0}(t) \geqslant & \lambda \int_{0}^{1} G_{1}(t, s)\left[f\left(s,\left[x_{0}(s)-\omega_{1}(s)\right]^{*},\left[y_{0}(s)-\omega_{2}(s)\right]^{*}\right)+q_{1}(s)\right] d s \\
> & \lambda v t^{\alpha-1} \int_{\xi}^{\eta}\left(1-(1-s)^{\alpha-1}\right) d s\left(\frac{1}{2} \lambda v \xi^{2(\alpha-1)} \gamma \int_{\xi}^{\eta}\left(1-(1-s)^{\alpha-1}\right) d s\right)^{-1} \\
& \cdot\left(\left(x_{0}(s)-\omega_{1}(s)\right)+y_{0}(s)-\omega_{2}(s)\right) \\
> & \lambda v \xi^{\alpha-1} \int_{\xi}^{\eta}\left(1-(1-s)^{\alpha-1}\right) d s\left(\frac{1}{2} \lambda v \xi^{2(\alpha-1)} \gamma \int_{\xi}^{\eta}\left(1-(1-s)^{\alpha-1}\right) d s\right)^{-1}\left(\frac{1}{2} \gamma \xi^{\alpha-1} R\right) \\
= & R, \quad t \in[\xi, \eta] .
\end{aligned}
$$

This is in contradiction with $\left(x_{0}, y_{0}\right) \in \partial P_{R}$. By Lemma 2.10 , for any $\lambda \in(0, \bar{\lambda})$, we have

$$
i\left(T, P_{R}, P\right)=0 \text {. }
$$

Similarly, (3.14) is valid if $\mathrm{g}_{\infty}=+\infty$. 
Step 3. We shall prove that there exist $\lambda^{*} \in(0, \bar{\lambda}]$ satisfying that for any $\lambda \in\left(0, \lambda^{*}\right)$, there exists $r^{\prime} \in(0, r)$ such that $i\left(T, P_{r^{\prime}}, P\right)=0$.

It follows from $\widehat{f}_{0} \geqslant L$ that there exists $\delta>0$ such that

$$
f(t, u, v) \geqslant L, \quad t \in[\xi, \eta], u+v \in(0, \delta) .
$$

Let

$$
\lambda^{*}=\min \left\{\frac{\gamma \delta}{2 \rho \int_{0}^{1}\left(q_{1}(s)+q_{2}(s)\right) d s}, \bar{\lambda}\right\} .
$$

For every $\lambda \in\left(0, \lambda^{*}\right)$, it is easy to see that

$$
\frac{\lambda \rho}{\gamma} \int_{0}^{1}\left(q_{1}(s)+q_{2}(s)\right) d s<\frac{\delta}{2}
$$

From $L>\frac{\int_{0}^{1}\left(q_{1}(s)+q_{2}(s)\right) d s}{\gamma^{2} \xi^{\alpha-1} \int_{\xi}^{\eta}\left(1-(1-s)^{\alpha-1}\right) d s}$, it follows that

$$
v \xi^{\alpha-1} L \int_{\xi}^{\eta}\left(1-(1-s)^{\alpha-1}\right) d s>\frac{\rho}{\gamma} \int_{0}^{1}\left(q_{1}(s)+q_{2}(s)\right) d s .
$$

So, we can choose $r^{\prime} \in\left(0, \frac{\delta}{2}\right)$ such that

$$
\lambda \frac{\rho}{\gamma} \int_{0}^{1}\left(q_{1}(s)+q_{2}(s)\right) d s<r^{\prime}<\lambda v \xi^{\alpha-1} L \int_{\xi}^{\eta}\left(1-(1-s)^{\alpha-1}\right) d s
$$

Now, we are ready to prove that

$$
\mathrm{T}(x, y) \neq \mu(x, y), \quad \forall(x, y) \in \partial \mathrm{P}_{\mathrm{r}^{\prime}}, \mu \in(0,1] .
$$

Suppose this is false. Then, there exist $\left(x_{0}, y_{0}\right) \in \partial P_{r^{\prime}}$ and $\mu_{0} \in(0,1]$ such that $T\left(x_{0}, y_{0}\right)=\mu_{0}\left(x_{0}, y_{0}\right)$, that is, $T_{1}\left(x_{0}, y_{0}\right)=\mu_{0} x_{0}, T_{2}\left(x_{0}, y_{0}\right)=\mu_{0} y_{0}$. Since $\left(x_{0}, y_{0}\right) \in P$, one has

$$
x_{0}(t) \geqslant \gamma t^{\alpha-1}\left\|\left(x_{0}, y_{0}\right)\right\|_{1}=\gamma t^{\alpha-1} r^{\prime}, \quad t \in[0,1] .
$$

This together with (2.12) and (3.16) implies

$$
x_{0}(t)-\omega_{1}(t) \geqslant t^{\alpha-1}\left(\gamma r^{\prime}-\lambda \rho \int_{0}^{1}\left(q_{1}(s)+q_{2}(s)\right) d s\right)>0, t \in(0,1] .
$$

In addition, $x_{0}(t)-\omega_{1}(t) \leqslant x_{0}(t) \leqslant\left\|x_{0}\right\| \leqslant\left\|\left(x_{0}, y_{0}\right)\right\|_{1}=r^{\prime}<\frac{\delta}{2}$. Similarly, we can get $0<y_{0}(t)-\omega_{2}(t)<\frac{\delta}{2}$ for $t \in(0,1]$, and then

$$
0<\left(x_{0}(t)-\omega_{1}(t)\right)+\left(y_{0}(t)-w_{2}(t)\right) \leqslant 2 r^{\prime}<\delta, \quad t \in(0,1] .
$$

Therefore, by (3.15) and (3.17), we have

$$
\begin{aligned}
x_{0}(t) \geqslant & T_{1}\left(x_{0}, y_{0}\right)(t) \\
= & \lambda \int_{0}^{1} G_{1}(t, s)\left[f\left(s,\left[x_{0}(s)-\omega_{1}(s)\right]^{*},\left[y_{0}(s)-\omega_{2}(s)\right]^{*}\right)+q_{1}(s)\right] d s \\
& +\lambda \int_{0}^{1} H_{1}(t, s)\left[g\left(s,\left[x_{0}(s)-\omega_{1}(s)\right]^{*},\left[y_{0}(s)-\omega_{2}(s)\right]^{*}\right)+q_{2}(s)\right] d s
\end{aligned}
$$




$$
\begin{aligned}
& \geqslant \lambda \int_{0}^{1} G_{1}(t, s)\left[f\left(s,\left[x_{0}(s)-\omega_{1}(s)\right]^{*},\left[y_{0}(s)-\omega_{2}(s)\right]^{*}\right)+q_{1}(s)\right] d s \\
& \geqslant \lambda \int_{\xi}^{\eta} G_{1}(t, s) f\left(s, x_{0}(s)-\omega_{1}(s), y_{0}(s)-\omega_{2}(s)\right) d s \\
& \geqslant \lambda v t^{\alpha-1} L \int_{\xi}^{\eta}\left(1-(1-s)^{\alpha-1}\right) d s \\
& \geqslant \lambda v \xi^{\alpha-1} L \int_{\xi}^{\eta}\left(1-(1-s)^{\alpha-1}\right) d s \\
& >r^{\prime}, \quad t \in[\xi, \eta] .
\end{aligned}
$$

Noticing $\left(x_{0}, y_{0}\right) \in \partial \mathrm{P}_{\mathrm{r}^{\prime}}$, we get a contradiction. By Lemma 2.10, we have

$$
i\left(T, P_{r^{\prime}}, P\right)=0 \text {. }
$$

Obviously, (3.18) is still valid if $\widehat{g}_{0} \geqslant$ L holds. Combining (3.18) with (3.11) and (3.14), we easily get

$$
\begin{aligned}
i\left(T, P_{R} \backslash \bar{P}_{r}, P\right) & =i\left(T, P_{R}, P\right)-i\left(T, P_{r}, P\right)=0-1=-1, \\
i\left(T, P_{r} \backslash \bar{P}_{r^{\prime}}, P\right) & =i\left(T, P_{r}, P\right)-i\left(T, P_{r^{\prime}}, P\right)=1-0=1 .
\end{aligned}
$$

Consequently, for any $\lambda \in\left(0, \lambda^{*}\right)$, BVP $(2.11)$ has at least two positive solutions $\left(x_{1}, y_{1}\right),\left(x_{2}, y_{2}\right)$ satisfying

$$
r^{\prime}<\left\|\left(x_{1}, y_{1}\right)\right\|_{1}<r<\left\|\left(x_{2}, y_{2}\right)\right\|_{1}<R
$$

Since $\left(x_{1}, y_{1}\right),\left(x_{2}, y_{2}\right) \in P$, one has

$$
\begin{aligned}
& x_{1}(t) \geqslant \gamma t^{\alpha-1}\left\|\left(x_{1}, y_{1}\right)\right\|_{1}>\gamma t^{\alpha-1} r^{\prime}>0, \quad t \in(0,1], \\
& x_{2}(t) \geqslant \gamma t^{\alpha-1}\left\|\left(x_{2}, y_{2}\right)\right\|_{1}>\gamma t^{\alpha-1} r>0, \quad t \in(0,1] .
\end{aligned}
$$

This together with (2.12) and (3.16) implies

$$
\begin{aligned}
& x_{1}(t)-\omega_{1}(t)>t^{\alpha-1}\left(\gamma r^{\prime}-\lambda \rho \int_{0}^{1}\left(q_{1}(s)+q_{2}(s)\right) d s\right)>0, t \in(0,1], \\
& x_{2}(t)-\omega_{1}(t)>t^{\alpha-1}\left(\gamma r-\lambda \rho \int_{0}^{1}\left(q_{1}(s)+q_{2}(s)\right) d s\right)>0, \quad t \in(0,1] .
\end{aligned}
$$

By a similar way, we also have $y_{1}(t)-\omega_{2}(t)>0, y_{2}(t)-\omega_{2}(t)>0, t \in(0,1]$.

Let $u_{1}(t)=x_{1}(t)-w_{1}(t), v_{1}(t)=y_{1}(t)-w_{2}(t)$ and $u_{2}(t)=x_{2}(t)-w_{1}(t), v_{2}(t)=y_{2}(t)-w_{2}(t)$. Then, $\left(u_{1}, v_{1}\right),\left(u_{2}, v_{2}\right)$ are two nonnegative solutions (positive on $\left.(0,1]\right)$ of the SBVP $(1.1)$.

\section{Examples}

Example 4.1. Consider the following SBVP of fractional differential equations:

$$
\left\{\begin{array}{l}
D_{0^{+}}^{\frac{7}{2}} u(t)+\lambda\left((u+v)^{2}-\frac{9}{2}(u+v)+\frac{7}{2}+2 \cos 2 \pi t\right)=0, t \in(0,1) \\
D_{0^{+}}^{\frac{7}{2}} v(t)+\lambda\left((u+v)^{4}-3(u+v)^{2}+2+\frac{1}{3} \sin 2 \pi t\right)=0, t \in(0,1) \\
u^{(j)}(0)=v^{(j)}(0)=0, j=0,1,2 \\
D_{0^{+}}^{\alpha-1} u(1)=12 \int_{0}^{\frac{3}{4}} v(t) d t \\
D_{0^{+}}^{\alpha-1} v(1)=3 \int_{0}^{\frac{2}{3}} u(t) d t .
\end{array}\right.
$$


Then there exists a constant $\lambda^{*}>0$ such that the SBVP (4.1) has at least two positive solutions for any $\lambda \in\left(0, \lambda^{*}\right)$.

Proof. (4.1) can be regarded as a SBVP of the form (1.1), where

$$
f(t, u, v)=(u+v)^{2}-\frac{9}{2}(u+v)+\frac{7}{2}+2 \cos 2 \pi t, \quad g(t, u, v)=(u+v)^{4}-3(u+v)^{2}+2+\frac{1}{3} \sin 2 \pi t,
$$

and $\alpha=\frac{7}{2}(\mathrm{n}=4), \lambda_{1}=12, \lambda_{2}=3, \eta_{1}=\frac{3}{4}, \eta_{2}=\frac{2}{3}, \lambda_{1} \lambda_{2}\left(\eta_{1} \eta_{2}\right)^{\alpha}=\frac{9}{4} \sqrt{2}<\frac{11025 \pi}{256}=\Gamma^{2}(\alpha+1)$. Take $\mathrm{q}_{1}(\mathrm{t})=$ $4, q_{2}(t)=2, p_{1}(t)=p_{2}(t)=1, h_{1}(u, v)=(u+v)^{2}-\frac{9}{2}(u+v)+10, h_{2}(u, v)=(u+v)^{4}-3(u+v)^{2}+5$, and then

$$
\begin{aligned}
& 0 \leqslant f(t, u, v)+q_{1}(t) \leqslant p_{1}(t) h_{1}(u, v), t \in(0,1), u, v \in[0,+\infty), \\
& 0 \leqslant g(t, u, v)+q_{2}(t) \leqslant p_{2}(t) h_{2}(u, v), t \in(0,1), u, v \in[0,+\infty)
\end{aligned}
$$

Obviously, $f(t, 0,0)=\frac{7}{2}+2 \cos 2 \pi t>0, g(t, 0,0)=2+\frac{1}{3} \sin 2 \pi t>0$. In addition, fix $[\xi, \eta] \subset(0,1)$, it is easy to see that $f_{\infty}=+\infty$ and $g_{\infty}=+\infty$. Thus, by Theorem 3.1, the conclusion follows.

Example 4.2. Consider the following SBVP of fractional differential equations:

$$
\left\{\begin{array}{l}
D_{0^{+}}^{\frac{5}{2}} u(t)+\lambda\left(\frac{16200}{\left(\frac{1}{10}+3(u+v)^{2}\right) \sqrt{t}}+\frac{1}{8} e^{2(u+v)}+\frac{2 \cos (\pi(u+v))}{\sqrt{t(1-t)}}\right)=0, t \in(0,1), \\
D_{0^{+}}^{\frac{5}{2}} v(t)+\lambda\left(\frac{15600}{\left(\frac{1}{6}+2(u+v)^{3}\right) \sqrt{t(1-t)}}+\frac{1}{10} e^{\frac{u+v}{4}}+\frac{\frac{1}{2} \cos \left(\frac{\pi}{4}(u+v)\right)}{\sqrt{1-t}}\right)=0, t \in(0,1), \\
u^{(j)}(0)=v^{(j)}(0)=0, j=0,1, \\
D_{0^{+}}^{\alpha-1} u(1)=6 \int_{0}^{\frac{2}{3}} v(t) d t, \\
D_{0^{+}}^{\alpha-1} v(1)=\frac{1}{2} \int_{0}^{\frac{3}{4}} u(t) d t .
\end{array}\right.
$$

Then there exists a constant $\lambda^{*}>0$ such that the SBVP (4.2) has at least two positive solutions for any $\lambda \in\left(0, \lambda^{*}\right)$.

Proof. (4.2) is a SBVP of type (1.1), where

$$
\begin{aligned}
& f(t, u, v)=\frac{16200}{\left(\frac{1}{10}+3(u+v)^{2}\right) \sqrt{t}}+\frac{1}{8} e^{2(u+v)}+\frac{2 \cos (\pi(u+v))}{\sqrt{t(1-t)}} \\
& g(t, u, v)=\frac{15600}{\left(\frac{1}{6}+2(u+v)^{3}\right) \sqrt{t(1-t)}}+\frac{1}{10} e^{\frac{u+v}{4}}+\frac{\frac{1}{2} \cos \left(\frac{\pi}{4}(u+v)\right)}{\sqrt{1-t}}
\end{aligned}
$$

and $\alpha=\frac{5}{2}(n=3), \lambda_{1}=6, \lambda_{2}=\frac{1}{2}, \eta_{1}=\frac{2}{3}, \eta_{2}=\frac{3}{4}, \lambda_{1} \lambda_{2}\left(\eta_{1} \eta_{2}\right)^{\alpha}=\frac{3}{8} \sqrt{2}<\frac{225 \pi}{64}=\Gamma^{2}(\alpha+1)$. Take

$$
q_{1}(t)=\frac{2}{\sqrt{t(1-t)}}, q_{2}(t)=\frac{1}{2 \sqrt{1-t}}, p_{1}(t)=\frac{1}{\sqrt{t(1-t)}}, p_{2}(t)=\frac{1}{\sqrt{t(1-t)}},
$$

and

$$
h_{1}(u, v)=\frac{1}{8} e^{2(u+v)}+\frac{16200}{\frac{1}{10}+3(u+v)^{2}}+4, \quad h_{2}(u, v)=\frac{1}{10} e^{\frac{u+v}{4}}+\frac{15600}{\frac{1}{6}+2(u+v)^{3}}+1,
$$

and then

$$
0 \leqslant f(t, u, v)+q_{1}(t) \leqslant p_{1}(t) h_{1}(u, v), t \in(0,1), u, v \in[0,+\infty),
$$




$$
0 \leqslant g(t, u, v)+q_{2}(t) \leqslant p_{2}(t) h_{2}(u, v), t \in(0,1), u, v \in[0,+\infty) .
$$

Let $[\xi, \eta]=\left[\frac{1}{2}, \frac{3}{4}\right]$. By direct calculation, we get

$$
\int_{0}^{1} p_{1}(s) d s=\int_{0}^{1} p_{2}(s) d s=\pi, \int_{0}^{1} q_{1}(s) d s=2 \pi, \int_{0}^{1} q_{2}(s) d s=1, \gamma=\frac{v}{\rho} \approx 0.024 \in(0,1),
$$

and

$$
\frac{\int_{0}^{1}\left(q_{1}(s)+q_{2}(s)\right) d s}{\gamma^{2} \xi^{\alpha-1} \int_{\xi}^{\eta}\left(1-(1-s)^{\alpha-1}\right) d s} \approx 186666
$$

and

$$
\widehat{\mathrm{f}}_{0} \approx 187066>186666, \widehat{\mathrm{g}}_{0} \approx 187200>186666 .
$$

In addition, it is easy to see that $f_{\infty}=+\infty$ and $g_{\infty}=+\infty$. Hence, our conclusion follows from Theorem 3.2.

\section{Acknowledgment}

The research was supported by the National Natural Science Foundation of China under grant 61503225, the Natural Science Fund for Distinguished Young Scholars of Shandong Province under grant JQ201613, and the Natural Science Foundation of Shandong Province under grant ZR2015FQ003.

\section{References}

[1] R. P. Agarwal, N. Hussain, M. A. Taoudi, Fixed point theorems in ordered Banach spaces and applications to nonlinear integral equations, Abstr. Appl. Anal., 2012 (2012), 15 pages. 1

[2] R. P. Agarwal, M. Meehan, D. O'Regan, Fixed point theory and applications, Cambridge Tracts in Mathematics, Cambridge University Press, Cambridge, (2001). 2.8

[3] R. P. Agarwal, D. O'Regan, A note on existence of nonnegative solutions to singular semi-positone problems, Nonlinear Anal., 36 (1999), 615-622. 1

[4] N. A. Asif, R. A. Khan, Positive solutions to singular system with four-point coupled boundary conditions, J. Math. Anal. Appl., 386 (2012), 848-861. 1

[5] A. Cabada, G.-T. Wang, Positive solutions of nonlinear fractional differential equations with integral boundary value conditions, J. Math. Anal. Appl., 389 (2012), 403-411. 1

[6] M.-Q. Feng, X.-M. Zhang, W.-G. Ge, New existence results for higher-order nonlinear fractional differential equation with integral boundary conditions, Bound. Value Probl., 2011 (2011), 20 pages. 1

[7] C. S. Goodrich, Existence of a positive solution to systems of differential equations of fractional order, Comput. Math. Appl., 62 (2011), 1251-1268. 1

[8] D. J. Guo, V. Lakshmikantham, Nonlinear problems in abstract cones, Notes and Reports in Mathematics in Science and Engineering, Academic Press, Inc., Boston, MA, (1988). 2.9, 2.10

[9] N. Hussain, M. A. Taoudi, Krasnosel'skii-type fixed point theorems with applications to Volterra integral equations, Fixed Point Theory Appl., 2013 (2013), 16 pages. 1

[10] M. Jia, X.-P. Liu, Multiplicity of solutions for integral boundary value problems of fractional differential equations with upper and lower solutions, Appl. Math. Comput., 232 (2014), 313-323. 1

[11] J.-Q. Jiang, L.-S. Liu, Y.-H. Wu, Positive solutions to singular fractional differential system with coupled boundary conditions, Commun. Nonlinear Sci. Numer. Simul., 18 (2013), 3061-3074. 1

[12] A. A. Kilbas, H. M. Srivastava, J. J. Trujillo, Theory and applications of fractional differential equations, North-Holland Mathematics Studies, Elsevier Science B.V., Amsterdam, (2006). 2.3

[13] V. Lakshmikantham, A. S. Vatsala, Basic theory of fractional differential equations, Nonlinear Anal., 69 (2008), $2677-$ 2682. 1

[14] K. Q. Lan, W. Lin, Multiple positive solutions of systems of Hammerstein integral equations with applications to fractional differential equations, J. Lond. Math. Soc., 83 (2011), 449-469. 1

[15] A. Leung, A semilinear reaction-diffusion prey-predator system with nonlinear coupled boundary conditions: equilibrium and stability, Indiana Univ. Math. J., 31 (1982), 223-241. 1

[16] Y. Li, Y.-Q. Chen, I. Podlubny, Stability of fractional-order nonlinear dynamic systems: Lyapunov direct method and generalized Mittag-Leffler stability, Comput. Math. Appl., 59 (2010), 1810-1821. 1

[17] S.-H. Liang, J.-H. Zhang, Positive solutions for boundary value problems of nonlinear fractional differential equation, Nonlinear Anal., 71 (2009), 5545-5550. 1 
[18] Y.-S. Liu, Twin solutions to singular semipositone problems, J. Math. Anal. Appl., 286 (2003), 248-260. 1

[19] Y.-S. Liu, B.-Q. Yan, Multiple solutions of singular boundary value problems for differential systems, J. Math. Anal. Appl., 287 (2003), 540-556. 1

[20] Y. Liu, W.-Q. Zhang, X.-P. Liu, A sufficient condition for the existence of a positive solution for a nonlinear fractional differential equation with the Riemann-Liouville derivative, Appl. Math. Lett., 25 (2012), 1986-1992. 1

[21] J.-X. Mao, Z.-Q. Zhao, N.-W. Xu, On existence and uniqueness of positive solutions for integral boundary value problems, Electron. J. Qual. Theory Differ. Equ., 2010 (2010), 8 pages. 1

[22] K. S. Miller, B. Ross, An introduction to the fractional calculus and fractional differential equations, A Wiley-Interscience Publication, John Wiley \& Sons, Inc., New York, (1993). 2

[23] S. K. Ntouyas, G.-T. Wang, L.-H. Zhang, Positive solutions of arbitrary order nonlinear fractional differential equations with advanced arguments, Opuscula Math., 31 (2011), 433-442.

[24] A. S. Perelson, D. E. Kirschner, R. De Boer, Dynamics of HIV infection of CD4 ${ }^{+}$T cells, Math. Biosci., 114 (1993), 81-125.

[25] I. Podlubny, Fractional differential equations, An introduction to fractional derivatives, fractional differential equations, to methods of their solution and some of their applications, Mathematics in Science and Engineering, Academic Press, Inc., San Diego, CA, (1999). 2

[26] S. G. Samko, A. A. Kilbas, O. I. Marichev, Fractional integrals and derivatives, Theory and applications, Edited and with a foreword by S. M. Nikol'skil, Translated from the 1987 Russian original, Revised by the authors, Gordon and Breach Science Publishers, Yverdon, (1993). 2

[27] M. Stojanović, R. Gorenflo, Nonlinear two-term time fractional diffusion-wave problem, Nonlinear Anal. Real World Appl., 11 (2010), 3512-3523. 1

[28] S. W. Vong, Positive solutions of singular fractional differential equations with integral boundary conditions, Math. Comput. Modelling, 57 (2013), 1053-1059. 1, 1

[29] J.-F. Xu, Z.-L. Wei, W. Dong, Uniqueness of positive solutions for a class of fractional boundary value problems, Appl. Math. Lett., 25 (2012), 590-593. 1

[30] W.-G. Yang, Positive solutions for a coupled system of nonlinear fractional differential equations with integral boundary conditions, Comput. Math. Appl., 63 (2012), 288-297. 1

[31] X.-J. Yang, Fractional derivatives of constant and variable orders applied to anomalous relaxation models in heat-transfer problems, Therm. Sci., (2016), 326-326. 1

[32] X.-J. Yang, H. M. Srivastava, J. A. Tenreiro Machado, A new fractional derivative without singular kernel: application to the modelling of the steady heat flow, Therm. Sci., 20 (2016), 753-756. 1

[33] X.-J. Yang, J. A. Tenreiro Machado, C. Cattani, F. Gao, On a fractal LC-electric circuit modeled by local fractional calculus, Commun. Nonlinear Sci. Numer. Simul., 47 (2017), 200-206. 1

[34] C.-J. Yuan, Multiple positive solutions for $(\mathrm{n}-1,1)$-type semipositone conjugate boundary value problems of nonlinear fractional differential equations, Electron. J. Qual. Theory Differ. Equ., 2010 (2010), 12 pages. 1

[35] C.-J. Yuan, Two positive solutions for $(n-1,1)$-type semipositone integral boundary value problems for coupled systems of nonlinear fractional differential equations, Commun. Nonlinear Sci. Numer. Simul., 17 (2012), 930-942. 1

[36] X.-U. Zhang, Y.-F. Han, Existence and uniqueness of positive solutions for higher order nonlocal fractional differential equations, Appl. Math. Lett., 25 (2012), 555-560. 1 